\title{
Mechanistic insights into the stereocontrolled synthesis of hexahydropyrrolo[2,3-b]indoles by electrophilic activation of tryptophan derivatives
}

\author{
Carlos Silva López, Carlos Pérez-Balado \\ Paula Rodríguez-Graña and Ángel R. de Lera
}

November 27, 2007

Departamento de Quimica Organica, Universidade de Vigo, Lagoas Marcosende, 36310, Vigo,
Galicia, Spain
${ }^{\dagger}$ Correspondence: qolera@uvigo.es 


\section{Contents}

1 Cartesian Coordinates $\quad$ S-3

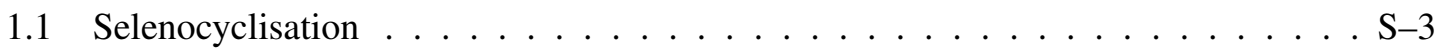

1.2 Bromocyclisation . . . . . . . . . . . . . . . S -16

2 General synthetic methods . . . . . . . . . . . . . . . . . . . . . . . . . . . . . . . S-28

2.1 Synthesis of the open-chain precursors . . . . . . . . . . . . . . . . . . . . . . . . . . . . S-32

2.2 Synthesis of 3a-bromo-hexahydropyrroloindoles . . . . . . . . . . . . . . . . . . . . S-32

3 1H-NMR, 13C-NMR Spectral Data and HPLC chromatograms . . . . . . . . . . . . . . . S-39 


\section{Cartesian Coordinates}

\subsection{Selenocyclisation}

Int- $1_{B}$

SCF Energy: -1112.43126447

Num. Imaginary Frequencies: 0 


$\begin{array}{lrrr}\mathrm{C} & -1.013405 & 1.615870 & -0.419452 \\ \mathrm{C} & -1.485586 & 2.874980 & -0.789883 \\ \mathrm{C} & -0.719008 & 3.603977 & -1.703402 \\ \mathrm{C} & 0.478402 & 3.105296 & -2.233338 \\ \mathrm{C} & 0.943881 & 1.848253 & -1.844818 \\ \mathrm{C} & 0.188219 & 1.108830 & -0.937072 \\ \mathrm{H} & -2.410497 & 3.261425 & -0.384272 \\ \mathrm{H} & -1.071428 & 4.582678 & -2.015366 \\ \mathrm{H} & 1.041618 & 3.695033 & -2.949202 \\ \mathrm{H} & 1.873103 & 1.459045 & -2.253787 \\ \mathrm{C} & 0.467640 & -0.221459 & -0.306051 \\ \mathrm{~N} & -1.578964 & 0.664735 & 0.454764 \\ \mathrm{C} & -0.910228 & -0.617341 & 0.290398 \\ \mathrm{C} & 1.338357 & -1.287154 & -1.032389 \\ \mathrm{H} & -0.835439 & -1.153689 & 1.235044 \\ \mathrm{C} & -2.676131 & 0.887734 & 1.260142 \\ \mathrm{O} & -3.275546 & 1.937322 & 1.347679 \\ \mathrm{O} & -2.984501 & -0.225899 & 1.973118 \\ \mathrm{H} & -3.767448 & 0.010721 & 2.503731 \\ \mathrm{C} & 2.362413 & -1.267496 & 0.129607 \\ \mathrm{H} & 1.744606 & -0.931661 & -1.980187 \\ \mathrm{H} & 0.838847 & -2.241469 & -1.191857 \\ \mathrm{C} & 3.825094 & -0.969221 & -0.148192 \\ \mathrm{H} & 2.333946 & -2.175508 & 0.747473 \\ \mathrm{~N} & 1.599235 & -0.172659 & 0.759738 \\ \mathrm{C} & 1.839370 & 0.590710 & 1.801279 \\ \mathrm{O} & 4.269328 & -1.566753 & -1.237906 \\ \mathrm{O} & 4.525258 & -0.290781 & 0.594722 \\ \mathrm{H} & 5.230893 & -1.401901 & -1.331149 \\ \mathrm{O} & 0.863567 & 1.346907 & 2.239953 \\ \mathrm{O} & 2.971500 & 0.636445 & 2.436707 \\ \mathrm{H} & 1.193905 & 1.950145 & 2.934443 \\ \mathrm{Se} & -1.941544 & -1.866640 & -0.976335 \\ \mathrm{H} & 3.721200 & 0.263967 & 1.857305 \\ \mathrm{C} & -2.497205 & -3.212316 & 0.403076 \\ \mathrm{H} & -3.170722 & -3.898109 & -0.114475 \\ \mathrm{H} & -1.632605 & -3.761362 & 0.778010 \\ & -3.027114 & -2.699989 & 1.204559\end{array}$


$\mathrm{P}-1_{B}$

SCF Energy: -1112.45639372

Num. Imaginary Frequencies: 0

$\begin{array}{lrrr}\mathrm{C} & 0.325913 & 0.373879 & 1.520364 \\ \mathrm{C} & 0.357313 & 0.372348 & 2.909772 \\ \mathrm{C} & 0.875340 & -0.765944 & 3.539244 \\ \mathrm{C} & 1.329788 & -1.863069 & 2.801444 \\ \mathrm{C} & 1.295609 & -1.840057 & 1.402683 \\ \mathrm{C} & 0.798757 & -0.706812 & 0.759886 \\ \mathrm{H} & 0.006342 & 1.224899 & 3.475940 \\ \mathrm{H} & 0.915274 & -0.794680 & 4.623858 \\ \mathrm{H} & 1.716865 & -2.737208 & 3.315755 \\ \mathrm{H} & 1.654400 & -2.692275 & 0.832698 \\ \mathrm{C} & 0.692732 & -0.386517 & -0.711330 \\ \mathrm{~N} & -0.174564 & 1.382917 & 0.641701 \\ \mathrm{C} & -0.279474 & 0.818302 & -0.678121 \\ \mathrm{C} & 0.013029 & -1.440689 & -1.590047 \\ \mathrm{H} & -0.157919 & 1.569998 & -1.454814 \\ \mathrm{C} & -0.091662 & 2.744561 & 0.924864 \\ \mathrm{O} & 0.102757 & 3.194178 & 2.029781 \\ \mathrm{O} & -0.271747 & 3.491276 & -0.188750 \\ \mathrm{H} & -0.177439 & 4.419965 & 0.092676 \\ \mathrm{C} & -1.491882 & -1.304409 & -1.283239 \\ \mathrm{H} & 0.371901 & -2.452435 & -1.397931 \\ \mathrm{H} & 0.182478 & -1.216439 & -2.645826 \\ \mathrm{C} & -2.088246 & -2.255867 & -0.240159 \\ \mathrm{H} & -2.088855 & -1.471874 & -2.189872 \\ \mathrm{~N} & -1.628153 & 0.120491 & -0.878019 \\ \mathrm{C} & -2.786061 & 0.752784 & -0.793735 \\ \mathrm{O} & -1.392224 & -3.356507 & -0.037837 \\ \mathrm{O} & -3.175982 & -2.043186 & 0.288545 \\ \mathrm{H} & -1.882530 & -3.934409 & 0.583747 \\ \mathrm{O} & -2.771407 & 2.060881 & -0.798127 \\ \mathrm{O} & -3.937458 & 0.162984 & -0.725295 \\ \mathrm{H} & -3.669466 & 2.404655 & -0.621878 \\ \mathrm{Se} & 2.441478 & 0.311478 & -1.525309 \\ \mathrm{H} & -3.825563 & -0.779651 & -0.332772 \\ \mathrm{C} & 3.741618 & -1.010070 & -0.781802 \\ \mathrm{H} & 4.719607 & -0.651072 & -1.109603 \\ \mathrm{H} & 3.693651 & -1.006100 & 0.306978 \\ \mathrm{H} & 3.561862 & -2.005119 & -1.189709\end{array}$


$\mathrm{B}_{1}$

SCF Energy: -1112.45717962

Num. Imaginary Frequencies: 0

$\begin{array}{lrrr}\mathrm{C} & -1.640967 & 1.402677 & -0.391815 \\ \mathrm{C} & -2.445204 & 2.535193 & -0.584796 \\ \mathrm{C} & -1.948210 & 3.515786 & -1.429933 \\ \mathrm{C} & -0.685553 & 3.419334 & -2.086811 \\ \mathrm{C} & 0.109435 & 2.317176 & -1.903800 \\ \mathrm{C} & -0.361319 & 1.279492 & -1.044962 \\ \mathrm{H} & -3.404009 & 2.629727 & -0.095130 \\ \mathrm{H} & -2.552519 & 4.401803 & -1.603475 \\ \mathrm{H} & -0.365274 & 4.227350 & -2.735732 \\ \mathrm{H} & 1.069906 & 2.226904 & -2.399872 \\ \mathrm{C} & 0.224103 & 0.089262 & -0.637129 \\ \mathrm{~N} & -1.847687 & 0.291244 & 0.383480 \\ \mathrm{C} & -0.756876 & -0.651224 & 0.197065 \\ \mathrm{C} & 1.584351 & -0.407689 & -0.980200 \\ \mathrm{H} & -0.367337 & -1.017161 & 1.145697 \\ \mathrm{C} & -2.922520 & 0.125156 & 1.273982 \\ \mathrm{O} & -3.832805 & 0.908532 & 1.382342 \\ \mathrm{O} & -2.771775 & -1.007415 & 1.980619 \\ \mathrm{H} & -3.539022 & -1.066762 & 2.581230 \\ \mathrm{C} & 2.588978 & -0.335796 & 0.224293 \\ \mathrm{H} & 1.996290 & 0.173989 & -1.809615 \\ \mathrm{H} & 1.521179 & -1.454216 & -1.295150 \\ \mathrm{C} & 3.942802 & -0.902039 & -0.216670 \\ \mathrm{H} & 2.200218 & -0.969716 & 1.030163 \\ \mathrm{~N} & 2.815575 & 1.001147 & 0.713384 \\ \mathrm{C} & 1.974946 & 1.539461 & 1.639597 \\ \mathrm{H} & 3.759654 & 1.363471 & 0.619328 \\ \mathrm{O} & 3.832326 & -2.183035 & -0.598732 \\ \mathrm{O} & 4.968743 & -0.263878 & -0.218418 \\ \mathrm{H} & 4.720163 & -2.492987 & -0.866668 \\ \mathrm{O} & 2.467039 & 2.680071 & 2.153789 \\ \mathrm{O} & 0.891001 & 1.052688 & 1.945575 \\ \mathrm{H} & 1.819292 & 3.004256 & 2.804719 \\ \mathrm{Se} & -1.342208 & -2.337943 & -0.854972 \\ \mathrm{C} & -1.163239 & -3.656334 & 0.643551 \\ \mathrm{H} & -1.537232 & -4.597269 & 0.233156 \\ \mathrm{H} & -0.117436 & -3.769295 & 0.929196 \\ \mathrm{H} & -1.784609 & -3.335199 & 1.477537\end{array}$


$\mathrm{B}_{2}$

SCF Energy: -1112.45156575

Num. Imaginary Frequencies: 0

$\begin{array}{lrrr}\mathrm{C} & -2.365277 & -0.121160 & -0.785536 \\ \mathrm{C} & -3.652539 & -0.352290 & -1.255719 \\ \mathrm{C} & -3.964987 & -1.672483 & -1.598953 \\ \mathrm{C} & -3.029596 & -2.707854 & -1.474073 \\ \mathrm{C} & -1.741838 & -2.454080 & -0.995593 \\ \mathrm{C} & -1.411870 & -1.144162 & -0.647426 \\ \mathrm{H} & -4.371474 & 0.448916 & -1.358952 \\ \mathrm{H} & -4.958696 & -1.893303 & -1.975769 \\ \mathrm{H} & -3.307821 & -3.718775 & -1.753930 \\ \mathrm{H} & -1.020758 & -3.259880 & -0.898681 \\ \mathrm{C} & -0.145569 & -0.553429 & -0.148660 \\ \mathrm{~N} & -1.737325 & 1.105499 & -0.384583 \\ \mathrm{C} & -0.452812 & 0.878914 & -0.034647 \\ \mathrm{C} & 1.164717 & -1.136716 & -0.672079 \\ \mathrm{H} & 0.253016 & 1.679577 & 0.172738 \\ \mathrm{C} & -2.387728 & 2.374900 & -0.331613 \\ \mathrm{O} & -3.536839 & 2.531387 & -0.647164 \\ \mathrm{O} & -1.533220 & 3.305233 & 0.101416 \\ \mathrm{H} & -2.007478 & 4.158553 & 0.117252 \\ \mathrm{C} & 2.507889 & -0.478715 & -0.259217 \\ \mathrm{H} & 1.101336 & -1.115584 & -1.767610 \\ \mathrm{H} & 1.181623 & -2.187454 & -0.368721 \\ \mathrm{C} & 3.646230 & -1.482909 & -0.503173 \\ \mathrm{H} & 2.501822 & -0.245933 & 0.811383 \\ \mathrm{~N} & 2.831873 & 0.723556 & -1.006696 \\ \mathrm{C} & 2.764473 & 1.953818 & -0.418962 \\ \mathrm{H} & 3.553824 & 0.595847 & -1.711953 \\ \mathrm{O} & 3.516272 & -2.569475 & 0.274965 \\ \mathrm{O} & 4.534587 & -1.311138 & -1.303663 \\ \mathrm{H} & 4.265917 & -3.165861 & 0.078989 \\ \mathrm{O} & 3.475544 & 2.864954 & -1.104793 \\ \mathrm{O} & 2.106644 & 2.210022 & 0.585525 \\ \mathrm{H} & 3.365500 & 3.717664 & -0.646996 \\ \mathrm{Se} & -0.106955 & -0.403658 & 2.007845 \\ \mathrm{C} & -1.971046 & -0.964249 & 2.440498 \\ \mathrm{H} & -1.963699 & -1.113640 & 3.523511 \\ \mathrm{H} & -2.672704 & -0.169976 & 2.184836 \\ \mathrm{H} & -2.209962 & -1.894536 & 1.926756\end{array}$


TS- $1_{B}$

SCF Energy: -1112.41375848

Num. Imaginary Frequencies: 1

$\begin{array}{lrrr}\mathrm{C} & -1.248673 & 1.582501 & -0.374193 \\ \mathrm{C} & -1.810917 & 2.837046 & -0.622532 \\ \mathrm{C} & -1.210394 & 3.612884 & -1.611395 \\ \mathrm{C} & -0.079740 & 3.180997 & -2.341409 \\ \mathrm{C} & 0.483612 & 1.945462 & -2.082355 \\ \mathrm{C} & -0.107168 & 1.131517 & -1.093218 \\ \mathrm{H} & -2.676323 & 3.179020 & -0.072153 \\ \mathrm{H} & -1.634096 & 4.588306 & -1.832588 \\ \mathrm{H} & 0.341762 & 3.825571 & -3.105373 \\ \mathrm{H} & 1.355129 & 1.605223 & -2.633099 \\ \mathrm{C} & 0.281784 & -0.135148 & -0.569101 \\ \mathrm{~N} & -1.631945 & 0.594582 & 0.527873 \\ \mathrm{C} & -0.873732 & -0.615332 & 0.266290 \\ \mathrm{C} & 1.358811 & -1.050175 & -1.137165 \\ \mathrm{H} & -0.615755 & -1.153614 & 1.173966 \\ \mathrm{C} & -2.664383 & 0.711137 & 1.452017 \\ \mathrm{O} & -3.339871 & 1.701584 & 1.605794 \\ \mathrm{O} & -2.788961 & -0.429703 & 2.165846 \\ \mathrm{H} & -3.523425 & -0.280126 & 2.790066 \\ \mathrm{C} & 2.379898 & -1.022591 & 0.024450 \\ \mathrm{H} & 1.774147 & -0.629528 & -2.055059 \\ \mathrm{H} & 0.972626 & -2.043543 & -1.371252 \\ \mathrm{C} & 3.848198 & -0.885387 & -0.367464 \\ \mathrm{H} & 2.329623 & -1.955606 & 0.608549 \\ \mathrm{~N} & 1.792399 & 0.080493 & 0.775747 \\ \mathrm{C} & 2.218327 & 0.716165 & 1.815469 \\ \mathrm{O} & 4.123642 & -1.460595 & -1.532841 \\ \mathrm{O} & 4.697510 & -0.356468 & 0.334467 \\ \mathrm{H} & 5.087862 & -1.398748 & -1.692952 \\ \mathrm{O} & 1.363708 & 1.532978 & 2.420553 \\ \mathrm{O} & 3.410355 & 0.661602 & 2.377443 \\ \mathrm{H} & 1.837161 & 2.022847 & 3.118067 \\ \mathrm{Se} & -1.944182 & -1.965383 & -0.890214 \\ \mathrm{H} & 4.064579 & 0.244759 & 1.742811 \\ \mathrm{C} & -2.150558 & -3.377176 & 0.519167 \\ \mathrm{H} & -2.810519 & -4.126465 & 0.076721 \\ \mathrm{H} & -1.187140 & -3.830287 & 0.754240 \\ \mathrm{H} & -2.618083 & -2.936876 & 1.398531\end{array}$


TS $-2 B$

SCF Energy: -1112.41418431

Num. Imaginary Frequencies: 1

$\begin{array}{lrrr}\mathrm{C} & -1.421708 & 1.171269 & 0.877349 \\ \mathrm{C} & -2.051927 & 2.196044 & 1.576687 \\ \mathrm{C} & -2.025949 & 3.463677 & 0.990629 \\ \mathrm{C} & -1.399856 & 3.707526 & -0.246047 \\ \mathrm{C} & -0.771377 & 2.679053 & -0.934946 \\ \mathrm{C} & -0.773913 & 1.395434 & -0.364123 \\ \mathrm{H} & -2.535894 & 2.013940 & 2.526424 \\ \mathrm{H} & -2.510417 & 4.285919 & 1.508648 \\ \mathrm{H} & -1.415670 & 4.708605 & -0.664372 \\ \mathrm{H} & -0.291159 & 2.857842 & -1.890246 \\ \mathrm{C} & -0.208621 & 0.142101 & -0.813941 \\ \mathrm{~N} & -1.289658 & -0.197955 & 1.202029 \\ \mathrm{C} & -0.652796 & -0.853904 & 0.159575 \\ \mathrm{C} & 0.983320 & -0.039104 & -1.716152 \\ \mathrm{H} & -0.156283 & -1.795316 & 0.342937 \\ \mathrm{C} & -1.826820 & -0.823377 & 2.342828 \\ \mathrm{O} & -2.379951 & -0.228838 & 3.232962 \\ \mathrm{O} & -1.622275 & -2.148815 & 2.276164 \\ \mathrm{H} & -1.986416 & -2.535867 & 3.094489 \\ \mathrm{C} & 2.331187 & -0.024750 & -0.925707 \\ \mathrm{H} & 0.985946 & 0.717295 & -2.502997 \\ \mathrm{H} & 0.937123 & -1.018175 & -2.196323 \\ \mathrm{C} & 2.887165 & 1.371707 & -0.612455 \\ \mathrm{H} & 3.083799 & -0.429075 & -1.627401 \\ \mathrm{~N} & 2.174651 & -0.885364 & 0.225132 \\ \mathrm{C} & 3.168356 & -1.305903 & 0.918577 \\ \mathrm{O} & 2.317647 & 2.372254 & -1.292709 \\ \mathrm{O} & 3.831586 & 1.555089 & 0.137510 \\ \mathrm{H} & 2.802945 & 3.192787 & -1.068997 \\ \mathrm{O} & 2.944141 & -2.291049 & 1.794384 \\ \mathrm{O} & 4.448024 & -0.921895 & 0.897738 \\ \mathrm{H} & 3.755628 & -2.424011 & 2.315730 \\ \mathrm{Se} & -2.021302 & -1.185451 & -1.582595 \\ \mathrm{H} & 4.487757 & 0.020021 & 0.590792 \\ \mathrm{C} & -1.189745 & -2.886465 & -2.233794 \\ \mathrm{H} & -1.960625 & -3.344855 & -2.859089 \\ \mathrm{H} & -0.307890 & -2.678115 & -2.840317 \\ \mathrm{H} & -0.956816 & -3.536236 & -1.390457\end{array}$


Int- $1_{A}$

SCF Energy: -1112.43155438

Num. Imaginary Frequencies: 0

$\begin{array}{lrrr}\mathrm{C} & 1.635559 & -1.283743 & -0.240366 \\ \mathrm{C} & 2.617214 & -2.255995 & -0.429622 \\ \mathrm{C} & 2.359386 & -3.251473 & -1.376394 \\ \mathrm{C} & 1.168569 & -3.284364 & -2.113468 \\ \mathrm{C} & 0.191091 & -2.309654 & -1.906066 \\ \mathrm{C} & 0.434227 & -1.312122 & -0.964068 \\ \mathrm{H} & 3.539481 & -2.229193 & 0.134350 \\ \mathrm{H} & 3.112785 & -4.014253 & -1.549201 \\ \mathrm{H} & 1.007918 & -4.065271 & -2.849566 \\ \mathrm{H} & -0.732304 & -2.331567 & -2.480047 \\ \mathrm{C} & -0.442787 & -0.188761 & -0.492912 \\ \mathrm{~N} & 1.632124 & -0.164689 & 0.620157 \\ \mathrm{C} & 0.548754 & 0.734126 & 0.254610 \\ \mathrm{C} & -1.524147 & 0.407890 & -1.438776 \\ \mathrm{H} & 0.114829 & 1.220394 & 1.126828 \\ \mathrm{C} & 2.598411 & 0.108322 & 1.565738 \\ \mathrm{O} & 3.551311 & -0.597511 & 1.814835 \\ \mathrm{O} & 2.322963 & 1.270917 & 2.211600 \\ \mathrm{H} & 3.051725 & 1.397254 & 2.846772 \\ \mathrm{C} & -2.627352 & -0.341579 & -0.652952 \\ \mathrm{H} & -1.424511 & 0.132525 & -2.488138 \\ \mathrm{H} & -1.596638 & 1.492824 & -1.359290 \\ \mathrm{C} & -3.859806 & 0.383748 & -0.145897 \\ \mathrm{H} & -2.976607 & -1.249646 & -1.163428 \\ \mathrm{~N} & -1.630925 & -0.676394 & 0.383660 \\ \mathrm{C} & -1.735817 & -1.353233 & 1.504142 \\ \mathrm{O} & -4.323776 & 1.277224 & -0.998272 \\ \mathrm{O} & -4.388650 & 0.115282 & 0.926992 \\ \mathrm{H} & -5.155438 & 1.660579 & -0.649107 \\ \mathrm{O} & -0.633952 & -1.749514 & 2.090219 \\ \mathrm{O} & -2.865664 & -1.644548 & 2.076984 \\ \mathrm{H} & -0.843079 & -2.165827 & 2.949451 \\ \mathrm{Se} & 1.182825 & 2.256914 & -0.973570 \\ \mathrm{H} & -3.614057 & -1.060864 & 1.710995 \\ \mathrm{C} & 0.880399 & 3.760785 & 0.317922 \\ \mathrm{H} & 1.302590 & 4.645758 & -0.162115 \\ \mathrm{H} & -0.185200 & 3.913480 & 0.493775 \\ \mathrm{H} & 1.414022 & 3.547829 & 1.243193\end{array}$


$\mathrm{P}-1_{A}$

SCF Energy: -1112.45512555

Num. Imaginary Frequencies: 0

$\begin{array}{lrrr}\mathrm{C} & 1.174590 & 1.033993 & 1.027981 \\ \mathrm{C} & 1.795025 & 1.742662 & 2.049640 \\ \mathrm{C} & 2.313826 & 1.003348 & 3.119886 \\ \mathrm{C} & 2.200107 & -0.388982 & 3.166893 \\ \mathrm{C} & 1.578819 & -1.085179 & 2.123611 \\ \mathrm{C} & 1.075478 & -0.364500 & 1.041660 \\ \mathrm{H} & 1.879738 & 2.820499 & 2.008856 \\ \mathrm{H} & 2.805235 & 1.529249 & 3.932719 \\ \mathrm{H} & 2.598346 & -0.935695 & 4.015816 \\ \mathrm{H} & 1.500699 & -2.168298 & 2.160653 \\ \mathrm{C} & 0.392759 & -0.842503 & -0.215515 \\ \mathrm{~N} & 0.532563 & 1.535027 & -0.145568 \\ \mathrm{C} & -0.235133 & 0.474187 & -0.737446 \\ \mathrm{C} & -0.772212 & -1.799946 & 0.002230 \\ \mathrm{H} & -0.317003 & 0.586051 & -1.815855 \\ \mathrm{C} & 0.977212 & 2.669220 & -0.822322 \\ \mathrm{O} & 1.699552 & 3.506159 & -0.333982 \\ \mathrm{O} & 0.496206 & 2.713263 & -2.086076 \\ \mathrm{H} & 0.875688 & 3.516061 & -2.488596 \\ \mathrm{C} & -1.885444 & -0.893079 & 0.566638 \\ \mathrm{H} & -0.540122 & -2.609883 & 0.695116 \\ \mathrm{H} & -1.091801 & -2.242887 & -0.944999 \\ \mathrm{C} & -3.268379 & -1.531292 & 0.472796 \\ \mathrm{H} & -1.702584 & -0.705770 & 1.634757 \\ \mathrm{~N} & -1.677317 & 0.386745 & -0.174221 \\ \mathrm{C} & -2.487072 & 1.435609 & -0.187146 \\ \mathrm{O} & -3.213393 & -2.834744 & 0.674217 \\ \mathrm{O} & -4.328352 & -0.936841 & 0.299131 \\ \mathrm{H} & -4.123948 & -3.197298 & 0.680371 \\ \mathrm{O} & -2.029052 & 2.545208 & -0.713290 \\ \mathrm{O} & -3.693969 & 1.473010 & 0.272924 \\ \mathrm{H} & -2.744564 & 3.210794 & -0.736706 \\ \mathrm{Se} & 1.686305 & -1.474291 & -1.674384 \\ \mathrm{H} & -4.100817 & 0.528644 & 0.355386 \\ \mathrm{C} & 2.925385 & -2.612112 & -0.596591 \\ \mathrm{H} & 3.704677 & -2.914587 & -1.299472 \\ \mathrm{H} & 3.364888 & -2.024034 & 0.208930 \\ \mathrm{H} & 2.412797 & -3.496359 & -0.216666\end{array}$


$\mathrm{A}_{1}$

SCF Energy: -1112.44412483

Num. Imaginary Frequencies: 0

$\begin{array}{lrrr}\mathrm{C} & 2.118701 & 0.877289 & 0.342444 \\ \mathrm{C} & 3.328333 & 1.498535 & 0.629190 \\ \mathrm{C} & 4.010099 & 1.045951 & 1.764428 \\ \mathrm{C} & 3.498429 & 0.020810 & 2.570230 \\ \mathrm{C} & 2.281052 & -0.592012 & 2.261270 \\ \mathrm{C} & 1.588001 & -0.156385 & 1.131836 \\ \mathrm{H} & 3.717116 & 2.296476 & 0.011308 \\ \mathrm{H} & 4.956563 & 1.508827 & 2.025341 \\ \mathrm{H} & 4.053633 & -0.301653 & 3.445185 \\ \mathrm{H} & 1.890241 & -1.389995 & 2.885810 \\ \mathrm{C} & 0.280652 & -0.578347 & 0.561081 \\ \mathrm{~N} & 1.182409 & 1.128791 & -0.716816 \\ \mathrm{C} & 0.113555 & 0.320248 & -0.592820 \\ \mathrm{C} & -0.857588 & -0.932352 & 1.541819 \\ \mathrm{H} & -0.767609 & 0.404501 & -1.213209 \\ \mathrm{C} & 1.372824 & 2.076292 & -1.769849 \\ \mathrm{O} & 2.337694 & 2.789293 & -1.835078 \\ \mathrm{O} & 0.339084 & 2.029484 & -2.612656 \\ \mathrm{H} & 0.492649 & 2.694171 & -3.311312 \\ \mathrm{C} & -2.270326 & -0.396884 & 1.222801 \\ \mathrm{H} & -0.565694 & -0.515120 & 2.513121 \\ \mathrm{H} & -0.911770 & -2.015694 & 1.680842 \\ \mathrm{C} & -2.857084 & -1.084451 & -0.019253 \\ \mathrm{H} & -2.914086 & -0.661016 & 2.070553 \\ \mathrm{~N} & -2.211745 & 1.046678 & 1.022944 \\ \mathrm{C} & -3.381872 & 1.692567 & 0.651725 \\ \mathrm{H} & -1.639270 & 1.564556 & 1.681299 \\ \mathrm{O} & -3.779274 & -1.985022 & 0.321033 \\ \mathrm{O} & -2.457761 & -0.908224 & -1.154015 \\ \mathrm{H} & -4.108355 & -2.404490 & -0.498608 \\ \mathrm{O} & -3.255529 & 3.025335 & 0.812661 \\ \mathrm{O} & -4.362206 & 1.125251 & 0.217689 \\ \mathrm{H} & -4.099324 & 3.424205 & 0.531755 \\ \mathrm{Se} & 0.517584 & -2.094858 & -0.947353 \\ \mathrm{C} & 2.506742 & -2.203312 & -1.045505 \\ \mathrm{H} & 2.697504 & -3.095109 & -1.649120 \\ \mathrm{H} & 2.912761 & -1.327101 & -1.551318 \\ \mathrm{H} & 2.927958 & -2.322289 & -0.048007\end{array}$


$\mathrm{A}_{2}$

SCF Energy: -1112.44920996

Num. Imaginary Frequencies: 0

$\begin{array}{lrrr}\mathrm{C} & -2.130776 & 1.112151 & 0.102925 \\ \mathrm{C} & -3.249295 & 1.931731 & 0.327980 \\ \mathrm{C} & -3.361399 & 3.063750 & -0.461375 \\ \mathrm{C} & -2.408150 & 3.418540 & -1.464592 \\ \mathrm{C} & -1.311252 & 2.630113 & -1.691428 \\ \mathrm{C} & -1.148440 & 1.449644 & -0.901424 \\ \mathrm{H} & -3.983001 & 1.680581 & 1.080686 \\ \mathrm{H} & -4.219117 & 3.713850 & -0.313673 \\ \mathrm{H} & -2.568346 & 4.318611 & -2.048462 \\ \mathrm{H} & -0.588710 & 2.883918 & -2.460156 \\ \mathrm{C} & -0.147236 & 0.487558 & -0.874486 \\ \mathrm{~N} & -1.741550 & -0.044420 & 0.721762 \\ \mathrm{C} & -0.536892 & -0.561439 & 0.093495 \\ \mathrm{C} & 1.113027 & 0.501172 & -1.685079 \\ \mathrm{H} & 0.254210 & -0.842828 & 0.796860 \\ \mathrm{C} & -2.399965 & -0.618182 & 1.829924 \\ \mathrm{O} & -3.426779 & -0.188505 & 2.292192 \\ \mathrm{O} & -1.707760 & -1.671408 & 2.284485 \\ \mathrm{H} & -2.194474 & -2.020650 & 3.055547 \\ \mathrm{C} & 2.426243 & 0.610780 & -0.850030 \\ \mathrm{H} & 1.079637 & 1.347628 & -2.376987 \\ \mathrm{H} & 1.168305 & -0.406457 & -2.297786 \\ \mathrm{C} & 2.819450 & -0.756228 & -0.268159 \\ \mathrm{H} & 3.226264 & 0.894829 & -1.543839 \\ \mathrm{~N} & 2.259297 & 1.581226 & 0.217799 \\ \mathrm{C} & 3.333934 & 1.784038 & 1.061827 \\ \mathrm{H} & 1.686176 & 2.395349 & 0.034022 \\ \mathrm{O} & 3.771318 & -1.323582 & -1.011718 \\ \mathrm{O} & 2.281209 & -1.294869 & 0.679278 \\ \mathrm{H} & 3.990651 & -2.190062 & -0.616513 \\ \mathrm{O} & 3.149076 & 2.886256 & 1.815975 \\ \mathrm{O} & 4.295008 & 1.043562 & 1.117970 \\ \mathrm{H} & 3.926874 & 2.961719 & 2.398198 \\ \mathrm{Se} & -0.893465 & -2.297940 & -0.994440 \\ \mathrm{C} & -0.037966 & -3.620998 & 0.247394 \\ \mathrm{H} & 0.011676 & -4.545111 & -0.333833 \\ \mathrm{H} & 0.962713 & -3.276103 & 0.505385 \\ \mathrm{H} & -0.663542 & -3.750358 & 1.127306\end{array}$


$\mathrm{TS}-1_{A}$

SCF Energy: -1112.41517238

Num. Imaginary Frequencies: 1

$\begin{array}{lrrr}\mathrm{C} & -1.478647 & 1.447399 & -0.290756 \\ \mathrm{C} & -2.238523 & 2.602304 & -0.489205 \\ \mathrm{C} & -1.848849 & 3.444561 & -1.529108 \\ \mathrm{C} & -0.739527 & 3.169611 & -2.358059 \\ \mathrm{C} & 0.019890 & 2.031484 & -2.147731 \\ \mathrm{C} & -0.351349 & 1.160661 & -1.105188 \\ \mathrm{H} & -3.093552 & 2.820248 & 0.135558 \\ \mathrm{H} & -2.429728 & 4.343801 & -1.712715 \\ \mathrm{H} & -0.488831 & 3.853604 & -3.161895 \\ \mathrm{H} & 0.873781 & 1.808915 & -2.781244 \\ \mathrm{C} & 0.262536 & -0.037217 & -0.614334 \\ \mathrm{~N} & -1.637311 & 0.419190 & 0.638810 \\ \mathrm{C} & -0.741109 & -0.669752 & 0.302503 \\ \mathrm{C} & 1.403786 & -0.793308 & -1.286891 \\ \mathrm{H} & -0.332073 & -1.173632 & 1.173860 \\ \mathrm{C} & -2.605623 & 0.380544 & 1.634409 \\ \mathrm{O} & -3.403486 & 1.262258 & 1.852783 \\ \mathrm{O} & -2.513226 & -0.771300 & 2.336383 \\ \mathrm{H} & -3.215074 & -0.733273 & 3.012574 \\ \mathrm{C} & 2.584217 & -0.072967 & -0.606563 \\ \mathrm{H} & 1.386038 & -0.711467 & -2.375768 \\ \mathrm{H} & 1.368959 & -1.853182 & -1.029791 \\ \mathrm{C} & 3.811848 & -0.894195 & -0.231903 \\ \mathrm{H} & 2.964941 & 0.743739 & -1.240049 \\ \mathrm{~N} & 1.829294 & 0.464862 & 0.526410 \\ \mathrm{C} & 2.247889 & 1.291751 & 1.430918 \\ \mathrm{O} & 3.956498 & -1.997875 & -0.953539 \\ \mathrm{O} & 4.609731 & -0.535578 & 0.622913 \\ \mathrm{H} & 4.793609 & -2.435844 & -0.694826 \\ \mathrm{O} & 1.338950 & 1.916366 & 2.164012 \\ \mathrm{O} & 3.498120 & 1.588346 & 1.723966 \\ \mathrm{H} & 1.789561 & 2.428229 & 2.861350 \\ \mathrm{Se} & -1.679146 & -2.147583 & -0.811991 \\ \mathrm{H} & 4.111639 & 0.893147 & 1.340101 \\ \mathrm{C} & -1.538620 & -3.603167 & 0.560414 \\ \mathrm{H} & -2.125571 & -4.431984 & 0.158944 \\ \mathrm{H} & -0.501596 & -3.914672 & 0.687400 \\ \mathrm{H} & -1.975248 & -3.252163 & 1.494462\end{array}$


TS- $2_{A}$

SCF Energy: -1112.41750539

Num. Imaginary Frequencies: 1

$\begin{array}{lrrr}\mathrm{C} & -1.729934 & -1.202061 & 0.589035 \\ \mathrm{C} & -2.508662 & -2.248287 & 1.074993 \\ \mathrm{C} & -2.366830 & -2.559345 & 2.429520 \\ \mathrm{C} & -1.486518 & -1.859350 & 3.276720 \\ \mathrm{C} & -0.717526 & -0.813901 & 2.784591 \\ \mathrm{C} & -0.837911 & -0.482311 & 1.424483 \\ \mathrm{H} & -3.183093 & -2.794079 & 0.429465 \\ \mathrm{H} & -2.958914 & -3.371539 & 2.840462 \\ \mathrm{H} & -1.415391 & -2.139110 & 4.322544 \\ \mathrm{H} & -0.047479 & -0.260543 & 3.435352 \\ \mathrm{C} & -0.152724 & 0.496958 & 0.617767 \\ \mathrm{~N} & -1.635472 & -0.671491 & -0.716112 \\ \mathrm{C} & -0.756990 & 0.408897 & -0.709904 \\ \mathrm{C} & 1.210809 & 1.052950 & 0.897660 \\ \mathrm{H} & -0.251992 & 0.685791 & -1.625104 \\ \mathrm{C} & -2.374231 & -1.106264 & -1.830905 \\ \mathrm{O} & -3.126533 & -2.047472 & -1.809328 \\ \mathrm{O} & -2.106389 & -0.323522 & -2.888970 \\ \mathrm{H} & -2.618018 & -0.675214 & -3.641676 \\ \mathrm{C} & 2.273333 & -0.008318 & 0.496977 \\ \mathrm{H} & 1.307186 & 1.315208 & 1.954512 \\ \mathrm{H} & 1.399042 & 1.951935 & 0.308784 \\ \mathrm{C} & 3.706880 & 0.479592 & 0.744692 \\ \mathrm{H} & 2.161297 & -0.887747 & 1.152378 \\ \mathrm{~N} & 2.042750 & -0.352032 & -0.895860 \\ \mathrm{C} & 2.613906 & -1.373552 & -1.425059 \\ \mathrm{O} & 3.813686 & 1.748244 & 1.142528 \\ \mathrm{O} & 4.674447 & -0.247063 & 0.582762 \\ \mathrm{H} & 4.764978 & 1.948687 & 1.259075 \\ \mathrm{O} & 2.185511 & -1.777542 & -2.625173 \\ \mathrm{O} & 3.589237 & -2.150292 & -0.944800 \\ \mathrm{H} & 2.783350 & -2.478003 & -2.941317 \\ \mathrm{Se} & -1.689046 & 2.245655 & 0.095426 \\ \mathrm{H} & 4.112576 & -1.651273 & -0.264589 \\ \mathrm{C} & -0.604924 & 3.565836 & -0.949439 \\ \mathrm{H} & -1.199288 & 4.483145 & -0.931916 \\ \mathrm{H} & 0.355834 & 3.743116 & -0.466101 \\ \mathrm{H} & -0.485199 & 3.218124 & -1.975474\end{array}$




\subsection{Bromocyclisation}

Int- $1_{B}$

SCF Energy: -1076.46747955

Num. Imaginary Frequencies: 0

$\begin{array}{lrrr}\mathrm{C} & -1.381807 & 1.228725 & -0.485347 \\ \mathrm{C} & -2.092283 & 2.330200 & -0.952637 \\ \mathrm{C} & -1.489343 & 3.099984 & -1.953934 \\ \mathrm{C} & -0.227464 & 2.784856 & -2.471475 \\ \mathrm{C} & 0.473808 & 1.678465 & -1.985918 \\ \mathrm{C} & -0.115279 & 0.904562 & -0.990019 \\ \mathrm{H} & -3.068845 & 2.572330 & -0.556364 \\ \mathrm{H} & -2.024770 & 3.960591 & -2.343388 \\ \mathrm{H} & 0.205063 & 3.398162 & -3.255167 \\ \mathrm{H} & 1.453064 & 1.431994 & -2.388396 \\ \mathrm{C} & 0.409274 & -0.295792 & -0.259159 \\ \mathrm{~N} & -1.739726 & 0.275374 & 0.502870 \\ \mathrm{C} & -0.858279 & -0.835426 & 0.452125 \\ \mathrm{C} & 1.409812 & -1.281259 & -0.930000 \\ \mathrm{H} & -0.704451 & -1.313729 & 1.413581 \\ \mathrm{C} & -2.855156 & 0.364049 & 1.332272 \\ \mathrm{O} & -3.647696 & 1.276933 & 1.313971 \\ \mathrm{O} & -2.904149 & -0.695736 & 2.164719 \\ \mathrm{H} & -3.717985 & -0.597970 & 2.692811 \\ \mathrm{C} & 2.437634 & -1.021945 & 0.200087 \\ \mathrm{H} & 1.742293 & -0.936519 & -1.909921 \\ \mathrm{H} & 1.060471 & -2.308579 & -1.009087 \\ \mathrm{C} & 3.844285 & -0.559859 & -0.135967 \\ \mathrm{H} & 2.533481 & -1.865546 & 0.897125 \\ \mathrm{~N} & 1.539724 & 0.016713 & 0.745630 \\ \mathrm{C} & 1.683935 & 0.904983 & 1.705338 \\ \mathrm{O} & 4.356929 & -1.201771 & -1.167426 \\ \mathrm{O} & 4.448705 & 0.280872 & 0.520307 \\ \mathrm{H} & 5.287317 & -0.922663 & -1.298846 \\ \mathrm{O} & 0.615741 & 1.552093 & 2.098718 \\ \mathrm{O} & 2.807566 & 1.171717 & 2.294729 \\ \mathrm{H} & 0.863144 & 2.258407 & 2.727947 \\ \mathrm{Br} & -1.676430 & -2.384249 & -0.697018 \\ \mathrm{H} & 3.594705 & 0.845495 & 1.733483\end{array}$


$\mathrm{P}-1_{B}$

SCF Energy: -1076.48929573

Num. Imaginary Frequencies: 0

$\begin{array}{lrrr}\mathrm{C} & 0.711079 & 0.212168 & 1.428807 \\ \mathrm{C} & 0.927623 & 0.162375 & 2.801989 \\ \mathrm{C} & 1.368427 & -1.053137 & 3.335768 \\ \mathrm{C} & 1.578771 & -2.177735 & 2.527478 \\ \mathrm{C} & 1.360203 & -2.108967 & 1.150708 \\ \mathrm{C} & 0.919571 & -0.903713 & 0.604356 \\ \mathrm{H} & 0.781023 & 1.036272 & 3.422475 \\ \mathrm{H} & 1.552629 & -1.120487 & 4.403679 \\ \mathrm{H} & 1.925680 & -3.104738 & 2.972735 \\ \mathrm{H} & 1.551849 & -2.969810 & 0.516787 \\ \mathrm{C} & 0.655374 & -0.536229 & -0.822481 \\ \mathrm{~N} & 0.265223 & 1.314642 & 0.636033 \\ \mathrm{C} & -0.074562 & 0.821665 & -0.672398 \\ \mathrm{C} & -0.273093 & -1.435468 & -1.634966 \\ \mathrm{H} & 0.104303 & 1.554818 & -1.453749 \\ \mathrm{C} & 0.605250 & 2.637628 & 0.925765 \\ \mathrm{O} & 0.990994 & 3.012153 & 2.007234 \\ \mathrm{O} & 0.430481 & 3.435386 & -0.151224 \\ \mathrm{H} & 0.708326 & 4.328385 & 0.125094 \\ \mathrm{C} & -1.685644 & -1.055076 & -1.152608 \\ \mathrm{H} & -0.063406 & -2.496055 & -1.496375 \\ \mathrm{H} & -0.187374 & -1.204536 & -2.699146 \\ \mathrm{C} & -2.307748 & -1.913596 & -0.044326 \\ \mathrm{H} & -2.402291 & -1.101470 & -1.983310 \\ \mathrm{~N} & -1.531107 & 0.364490 & -0.731116 \\ \mathrm{C} & -2.551518 & 1.180464 & -0.519014 \\ \mathrm{O} & -1.768894 & -3.106783 & 0.095538 \\ \mathrm{O} & -3.287580 & -1.535880 & 0.591671 \\ \mathrm{H} & -2.270412 & -3.610548 & 0.770718 \\ \mathrm{O} & -2.316509 & 2.466128 & -0.520152 \\ \mathrm{O} & -3.770055 & 0.786808 & -0.330332 \\ \mathrm{H} & -3.119963 & 2.954009 & -0.250427 \\ \mathrm{Br} & 2.412253 & -0.265558 & -1.852110 \\ \mathrm{H} & -3.777946 & -0.170023 & 0.044635 \\ & & & \end{array}$


$\mathrm{B}_{1}$

SCF Energy: -1076.48335659

Num. Imaginary Frequencies: 0

$\begin{array}{lrrr}\mathrm{C} & -1.936965 & 0.932585 & -0.387822 \\ \mathrm{C} & -2.967421 & 1.837557 & -0.655591 \\ \mathrm{C} & -2.702615 & 2.821825 & -1.601112 \\ \mathrm{C} & -1.457044 & 2.939361 & -2.282660 \\ \mathrm{C} & -0.438753 & 2.057059 & -2.021910 \\ \mathrm{C} & -0.665575 & 1.029729 & -1.058624 \\ \mathrm{H} & -3.920086 & 1.767844 & -0.149623 \\ \mathrm{H} & -3.487767 & 3.535311 & -1.835350 \\ \mathrm{H} & -1.325867 & 3.731994 & -3.011280 \\ \mathrm{H} & 0.514000 & 2.130369 & -2.535541 \\ \mathrm{C} & 0.167926 & 0.035588 & -0.566668 \\ \mathrm{~N} & -1.894888 & -0.126927 & 0.491240 \\ \mathrm{C} & -0.642303 & -0.815129 & 0.366045 \\ \mathrm{C} & 1.576820 & -0.245418 & -0.951727 \\ \mathrm{H} & -0.196366 & -1.082969 & 1.316533 \\ \mathrm{C} & -2.900957 & -0.443203 & 1.429979 \\ \mathrm{O} & -3.955510 & 0.136360 & 1.492893 \\ \mathrm{O} & -2.490004 & -1.450714 & 2.207981 \\ \mathrm{H} & -3.212716 & -1.654618 & 2.831500 \\ \mathrm{C} & 2.601948 & -0.148191 & 0.228597 \\ \mathrm{H} & 1.889910 & 0.438175 & -1.745421 \\ \mathrm{H} & 1.635505 & -1.267543 & -1.343808 \\ \mathrm{C} & 3.987209 & -0.597746 & -0.253609 \\ \mathrm{H} & 2.283433 & -0.840978 & 1.018010 \\ \mathrm{~N} & 2.749807 & 1.185031 & 0.755463 \\ \mathrm{C} & 1.797054 & 1.722613 & 1.553959 \\ \mathrm{H} & 3.675437 & 1.600045 & 0.702465 \\ \mathrm{O} & 3.952579 & -1.851974 & -0.722509 \\ \mathrm{O} & 4.970446 & 0.102410 & -0.205402 \\ \mathrm{H} & 4.857021 & -2.095791 & -1.003252 \\ \mathrm{O} & 2.184165 & 2.885592 & 2.097398 \\ \mathrm{O} & 0.693307 & 1.207123 & 1.739501 \\ \mathrm{H} & 1.452568 & 3.206868 & 2.654431 \\ \mathrm{Br} & -0.900152 & -2.624732 & -0.608504\end{array}$


$\mathrm{B}_{2}$

SCF Energy: -1076.47171814

Num. Imaginary Frequencies: 0

$\begin{array}{lrrr}\mathrm{C} & -2.472852 & -0.071046 & -0.558425 \\ \mathrm{C} & -3.779037 & -0.224622 & -0.999746 \\ \mathrm{C} & -4.151471 & -1.517493 & -1.392878 \\ \mathrm{C} & -3.250840 & -2.586120 & -1.344668 \\ \mathrm{C} & -1.937603 & -2.402650 & -0.894316 \\ \mathrm{C} & -1.553443 & -1.128244 & -0.495238 \\ \mathrm{H} & -4.472003 & 0.604109 & -1.044646 \\ \mathrm{H} & -5.164207 & -1.685337 & -1.744764 \\ \mathrm{H} & -3.574231 & -3.573646 & -1.657380 \\ \mathrm{H} & -1.249221 & -3.240302 & -0.843911 \\ \mathrm{C} & -0.250127 & -0.601365 & 0.007832 \\ \mathrm{~N} & -1.779424 & 1.116456 & -0.111369 \\ \mathrm{C} & -0.526071 & 0.850743 & 0.190106 \\ \mathrm{C} & 1.022360 & -1.072993 & -0.695645 \\ \mathrm{H} & 0.196152 & 1.598109 & 0.528927 \\ \mathrm{C} & -2.403084 & 2.424114 & 0.033944 \\ \mathrm{O} & -3.549574 & 2.612706 & -0.251893 \\ \mathrm{O} & -1.509988 & 3.286921 & 0.502203 \\ \mathrm{H} & -1.943808 & 4.157195 & 0.601607 \\ \mathrm{C} & 2.361094 & -0.389743 & -0.307092 \\ \mathrm{H} & 0.862160 & -0.930399 & -1.771958 \\ \mathrm{H} & 1.093777 & -2.150031 & -0.519168 \\ \mathrm{C} & 3.523961 & -1.337318 & -0.645385 \\ \mathrm{H} & 2.381033 & -0.206074 & 0.772352 \\ \mathrm{~N} & 2.604037 & 0.857377 & -1.014350 \\ \mathrm{C} & 2.577506 & 2.058643 & -0.361760 \\ \mathrm{H} & 3.286771 & 0.776849 & -1.765087 \\ \mathrm{O} & 3.456337 & -2.468510 & 0.072733 \\ \mathrm{O} & 4.375935 & -1.087935 & -1.464884 \\ \mathrm{H} & 4.218709 & -3.027509 & -0.177207 \\ \mathrm{O} & 3.255498 & 2.997794 & -1.041570 \\ \mathrm{O} & 1.977038 & 2.272525 & 0.687634 \\ \mathrm{H} & 3.178176 & 3.829173 & -0.539767 \\ \mathrm{Br} & -0.102569 & -0.996812 & 2.079625\end{array}$


TS- $1_{B}$

SCF Energy: -1076.44368955

Num. Imaginary Frequencies: 1

$\begin{array}{lrrr}\mathrm{C} & -1.702045 & 1.150850 & -0.373956 \\ \mathrm{C} & -2.565655 & 2.213595 & -0.627890 \\ \mathrm{C} & -2.239024 & 3.044898 & -1.700098 \\ \mathrm{C} & -1.092852 & 2.848091 & -2.505936 \\ \mathrm{C} & -0.232720 & 1.800567 & -2.244719 \\ \mathrm{C} & -0.536949 & 0.934171 & -1.168407 \\ \mathrm{H} & -3.446386 & 2.377723 & -0.022783 \\ \mathrm{H} & -2.898833 & 3.877493 & -1.926797 \\ \mathrm{H} & -0.896395 & 3.526689 & -3.328929 \\ \mathrm{H} & 0.651969 & 1.639884 & -2.852372 \\ \mathrm{C} & 0.162590 & -0.172372 & -0.645041 \\ \mathrm{~N} & -1.763845 & 0.162368 & 0.608090 \\ \mathrm{C} & -0.748005 & -0.808956 & 0.373936 \\ \mathrm{C} & 1.394111 & -0.839409 & -1.216344 \\ \mathrm{H} & -0.316363 & -1.233104 & 1.271096 \\ \mathrm{C} & -2.716542 & 0.099189 & 1.636783 \\ \mathrm{O} & -3.612623 & 0.895206 & 1.772111 \\ \mathrm{O} & -2.462512 & -0.954813 & 2.428456 \\ \mathrm{H} & -3.158269 & -0.975556 & 3.112052 \\ \mathrm{C} & 2.406588 & -0.680120 & -0.060252 \\ \mathrm{H} & 1.724853 & -0.302789 & -2.107590 \\ \mathrm{H} & 1.204619 & -1.876485 & -1.502239 \\ \mathrm{C} & 3.856254 & -0.439363 & -0.477537 \\ \mathrm{H} & 2.439581 & -1.600612 & 0.545751 \\ \mathrm{~N} & 1.752945 & 0.394607 & 0.680923 \\ \mathrm{C} & 2.149603 & 0.998607 & 1.750820 \\ \mathrm{O} & 4.142737 & -0.946146 & -1.671926 \\ \mathrm{O} & 4.684187 & 0.112881 & 0.231084 \\ \mathrm{H} & 5.097452 & -0.815264 & -1.847315 \\ \mathrm{O} & 1.258483 & 1.743441 & 2.397271 \\ \mathrm{O} & 3.344366 & 0.980296 & 2.312281 \\ \mathrm{H} & 1.712523 & 2.221877 & 3.115262 \\ \mathrm{Br} & -1.534508 & -2.483148 & -0.609858 \\ \mathrm{H} & 4.020122 & 0.626097 & 1.663437\end{array}$


TS $-2_{B}$

SCF Energy: -1076.43803638

Num. Imaginary Frequencies: 1

$\begin{array}{lrrr}\mathrm{C} & 1.325342 & -0.520672 & 1.235244 \\ \mathrm{C} & 1.839232 & -1.020534 & 2.419952 \\ \mathrm{C} & 1.497910 & -2.342500 & 2.740477 \\ \mathrm{C} & 0.677962 & -3.135620 & 1.916145 \\ \mathrm{C} & 0.167088 & -2.624925 & 0.731794 \\ \mathrm{C} & 0.489956 & -1.297753 & 0.386312 \\ \mathrm{H} & 2.468956 & -0.421865 & 3.063637 \\ \mathrm{H} & 1.886673 & -2.767416 & 3.661152 \\ \mathrm{H} & 0.452689 & -4.155171 & 2.210352 \\ \mathrm{H} & -0.460596 & -3.225390 & 0.083565 \\ \mathrm{C} & 0.122822 & -0.494489 & -0.730046 \\ \mathrm{~N} & 1.482052 & 0.756913 & 0.640730 \\ \mathrm{C} & 0.833169 & 0.780152 & -0.557757 \\ \mathrm{C} & -1.032630 & -0.657914 & -1.673617 \\ \mathrm{H} & 0.610815 & 1.711973 & -1.050938 \\ \mathrm{C} & 2.241130 & 1.826989 & 1.184894 \\ \mathrm{O} & 2.789885 & 1.763044 & 2.252772 \\ \mathrm{O} & 2.230070 & 2.861164 & 0.338887 \\ \mathrm{H} & 2.754328 & 3.579718 & 0.741175 \\ \mathrm{C} & -2.297324 & -0.037478 & -1.001983 \\ \mathrm{H} & -1.183096 & -1.704509 & -1.941616 \\ \mathrm{H} & -0.826480 & -0.102576 & -2.590473 \\ \mathrm{C} & -3.216524 & -1.030537 & -0.277356 \\ \mathrm{H} & -2.939079 & 0.355174 & -1.808341 \\ \mathrm{~N} & -1.817048 & 1.031003 & -0.137698 \\ \mathrm{C} & -2.596141 & 1.930781 & 0.349945 \\ \mathrm{O} & -2.891718 & -2.320483 & -0.402285 \\ \mathrm{O} & -4.208820 & -0.664437 & 0.330988 \\ \mathrm{H} & -3.586235 & -2.845658 & 0.046464 \\ \mathrm{O} & -2.032244 & 2.991444 & 0.929987 \\ \mathrm{O} & -3.926447 & 1.982935 & 0.347087 \\ \mathrm{H} & -2.735258 & 3.529679 & 1.336268 \\ \mathrm{Br} & 2.119812 & -0.248337 & -2.236490 \\ \mathrm{H} & -4.288934 & 1.060075 & 0.281177\end{array}$


Int- $1_{A}$

SCF Energy: -1076.46776983

Num. Imaginary Frequencies: 0

$\begin{array}{lrrr}\mathrm{C} & 1.785317 & -0.938071 & -0.274264 \\ \mathrm{C} & 2.874861 & -1.766012 & -0.525489 \\ \mathrm{C} & 2.736328 & -2.708841 & -1.550517 \\ \mathrm{C} & 1.559099 & -2.820329 & -2.299031 \\ \mathrm{C} & 0.473516 & -1.983002 & -2.029418 \\ \mathrm{C} & 0.597691 & -1.043985 & -1.009631 \\ \mathrm{H} & 3.787132 & -1.676200 & 0.048095 \\ \mathrm{H} & 3.573573 & -3.363046 & -1.774038 \\ \mathrm{H} & 1.490709 & -3.554742 & -3.094874 \\ \mathrm{H} & -0.438498 & -2.065352 & -2.615572 \\ \mathrm{C} & -0.395934 & -0.050086 & -0.477129 \\ \mathrm{~N} & 1.642925 & 0.105154 & 0.679391 \\ \mathrm{C} & 0.488709 & 0.876150 & 0.388667 \\ \mathrm{C} & -1.496263 & 0.538643 & -1.407111 \\ \mathrm{H} & 0.020088 & 1.311939 & 1.264683 \\ \mathrm{C} & 2.560049 & 0.401056 & 1.684490 \\ \mathrm{O} & 3.586585 & -0.208811 & 1.873457 \\ \mathrm{O} & 2.123624 & 1.442252 & 2.423519 \\ \mathrm{H} & 2.818733 & 1.625984 & 3.082159 \\ \mathrm{C} & -2.561196 & -0.330108 & -0.693788 \\ \mathrm{H} & -1.356002 & 0.332107 & -2.467318 \\ \mathrm{H} & -1.646271 & 1.608656 & -1.264854 \\ \mathrm{C} & -3.832321 & 0.285568 & -0.136537 \\ \mathrm{H} & -2.859444 & -1.213881 & -1.274262 \\ \mathrm{~N} & -1.550556 & -0.687994 & 0.323073 \\ \mathrm{C} & -1.621530 & -1.456233 & 1.388204 \\ \mathrm{O} & -4.335051 & 1.227688 & -0.908078 \\ \mathrm{O} & -4.349780 & -0.108224 & 0.903108 \\ \mathrm{H} & -5.184594 & 1.542674 & -0.533828 \\ \mathrm{O} & -0.501182 & -1.826508 & 1.954532 \\ \mathrm{O} & -2.735816 & -1.859363 & 1.916308 \\ \mathrm{H} & -0.687775 & -2.321570 & 2.776840 \\ \mathrm{Br} & 0.972436 & 2.565110 & -0.754463 \\ \mathrm{H} & -3.516507 & -1.290312 & 1.588481 \\ & & & \end{array}$


$\mathrm{P}-1_{A}$

SCF Energy: -1076.48815250

Num. Imaginary Frequencies: 0

$\begin{array}{lrrr}\mathrm{C} & 1.553499 & 0.548605 & 0.939691 \\ \mathrm{C} & 2.404543 & 1.032997 & 1.926986 \\ \mathrm{C} & 2.820846 & 0.128683 & 2.910288 \\ \mathrm{C} & 2.397449 & -1.206170 & 2.908689 \\ \mathrm{C} & 1.547468 & -1.676583 & 1.906020 \\ \mathrm{C} & 1.125968 & -0.786077 & 0.919289 \\ \mathrm{H} & 2.742100 & 2.060671 & 1.919207 \\ \mathrm{H} & 3.489257 & 0.475934 & 3.692258 \\ \mathrm{H} & 2.739877 & -1.881995 & 3.685648 \\ \mathrm{H} & 1.244816 & -2.719794 & 1.883579 \\ \mathrm{C} & 0.250611 & -1.016066 & -0.271689 \\ \mathrm{~N} & 0.977063 & 1.245798 & -0.166461 \\ \mathrm{C} & -0.052994 & 0.427973 & -0.741922 \\ \mathrm{C} & -1.090121 & -1.685011 & -0.025052 \\ \mathrm{H} & -0.151232 & 0.576010 & -1.813140 \\ \mathrm{C} & 1.639315 & 2.278098 & -0.833428 \\ \mathrm{O} & 2.559890 & 2.901455 & -0.361443 \\ \mathrm{O} & 1.115666 & 2.486609 & -2.062015 \\ \mathrm{H} & 1.647231 & 3.197960 & -2.464906 \\ \mathrm{C} & -1.932745 & -0.559014 & 0.603829 \\ \mathrm{H} & -1.018095 & -2.547854 & 0.637822 \\ \mathrm{H} & -1.540326 & -2.010101 & -0.966164 \\ \mathrm{C} & -3.435007 & -0.835378 & 0.529566 \\ \mathrm{H} & -1.691089 & -0.461331 & 1.672171 \\ \mathrm{~N} & -1.442065 & 0.665861 & -0.100092 \\ \mathrm{C} & -1.960349 & 1.883763 & -0.001196 \\ \mathrm{O} & -3.698380 & -2.126375 & 0.576775 \\ \mathrm{O} & -4.315491 & 0.019143 & 0.502381 \\ \mathrm{H} & -4.669373 & -2.258996 & 0.605300 \\ \mathrm{O} & -1.271738 & 2.876585 & -0.505722 \\ \mathrm{O} & -3.087453 & 2.185428 & 0.551337 \\ \mathrm{H} & -1.794685 & 3.700265 & -0.440403 \\ \mathrm{Br} & 1.255459 & -2.023966 & -1.754504 \\ \mathrm{H} & -3.715410 & 1.368753 & 0.625223\end{array}$


$\mathrm{A}_{1}$

SCF Energy: -1076.46345281

Num. Imaginary Frequencies: 0

$\begin{array}{lrrr}\mathrm{C} & -2.084479 & 0.711494 & -0.191338 \\ \mathrm{C} & -3.271601 & 1.370726 & -0.474011 \\ \mathrm{C} & -4.071721 & 0.806616 & -1.477285 \\ \mathrm{C} & -3.686324 & -0.354149 & -2.155215 \\ \mathrm{C} & -2.482313 & -0.999346 & -1.849329 \\ \mathrm{C} & -1.678574 & -0.456052 & -0.852766 \\ \mathrm{H} & -3.564184 & 2.271970 & 0.047018 \\ \mathrm{H} & -5.010227 & 1.289023 & -1.730709 \\ \mathrm{H} & -4.330966 & -0.764310 & -2.925806 \\ \mathrm{H} & -2.198164 & -1.911456 & -2.365347 \\ \mathrm{C} & -0.356120 & -0.894610 & -0.307082 \\ \mathrm{~N} & -1.048307 & 1.041294 & 0.760842 \\ \mathrm{C} & -0.040946 & 0.192477 & 0.674391 \\ \mathrm{C} & 0.738242 & -1.324236 & -1.318075 \\ \mathrm{H} & 0.835174 & 0.225494 & 1.311382 \\ \mathrm{C} & -1.112193 & 2.144115 & 1.695779 \\ \mathrm{O} & -2.037587 & 2.904224 & 1.722301 \\ \mathrm{O} & -0.020671 & 2.144576 & 2.455203 \\ \mathrm{H} & -0.085854 & 2.888511 & 3.085402 \\ \mathrm{C} & 2.034523 & -0.489367 & -1.228525 \\ \mathrm{H} & 0.316390 & -1.196773 & -2.320608 \\ \mathrm{H} & 0.976505 & -2.384146 & -1.213167 \\ \mathrm{C} & 2.887432 & -0.923369 & -0.022847 \\ \mathrm{H} & 2.628291 & -0.675805 & -2.130044 \\ \mathrm{~N} & 1.656961 & 0.918227 & -1.106013 \\ \mathrm{C} & 2.689819 & 1.851578 & -0.936674 \\ \mathrm{H} & 0.955087 & 1.229904 & -1.772811 \\ \mathrm{O} & 3.843040 & -1.766532 & -0.415222 \\ \mathrm{O} & 2.669490 & -0.619475 & 1.131065 \\ \mathrm{H} & 4.339677 & -2.053446 & 0.377026 \\ \mathrm{O} & 2.209380 & 3.097335 & -1.108586 \\ \mathrm{O} & 3.828376 & 1.559111 & -0.656600 \\ \mathrm{H} & 2.959161 & 3.711793 & -1.000621 \\ \mathrm{Br} & -0.668919 & -2.420474 & 1.105344\end{array}$


$\mathrm{A}_{2}$

SCF Energy: -1076.47088619

Num. Imaginary Frequencies: 0

$\begin{array}{lrrr}\mathrm{C} & -2.092745 & 0.985561 & 0.112340 \\ \mathrm{C} & -3.184997 & 1.834438 & 0.333763 \\ \mathrm{C} & -3.268630 & 2.962263 & -0.469244 \\ \mathrm{C} & -2.310903 & 3.283570 & -1.480842 \\ \mathrm{C} & -1.235745 & 2.465778 & -1.702892 \\ \mathrm{C} & -1.099969 & 1.289808 & -0.897497 \\ \mathrm{H} & -3.923501 & 1.610037 & 1.090613 \\ \mathrm{H} & -4.109492 & 3.635247 & -0.326208 \\ \mathrm{H} & -2.451908 & 4.181427 & -2.072788 \\ \mathrm{H} & -0.508431 & 2.691376 & -2.475887 \\ \mathrm{C} & -0.128694 & 0.305177 & -0.869183 \\ \mathrm{~N} & -1.736188 & -0.177976 & 0.742335 \\ \mathrm{C} & -0.539158 & -0.710725 & 0.146386 \\ \mathrm{C} & 1.125541 & 0.241067 & -1.681735 \\ \mathrm{H} & 0.237185 & -1.022085 & 0.850408 \\ \mathrm{C} & -2.398698 & -0.730897 & 1.869694 \\ \mathrm{O} & -3.418477 & -0.276255 & 2.320398 \\ \mathrm{O} & -1.711915 & -1.782749 & 2.316675 \\ \mathrm{H} & -2.191796 & -2.152933 & 3.082252 \\ \mathrm{C} & 2.439082 & 0.326282 & -0.844227 \\ \mathrm{H} & 1.123703 & 1.062414 & -2.404233 \\ \mathrm{H} & 1.146061 & -0.689728 & -2.261311 \\ \mathrm{C} & 2.748023 & -1.017821 & -0.160533 \\ \mathrm{H} & 3.257812 & 0.515809 & -1.548499 \\ \mathrm{~N} & 2.314159 & 1.376517 & 0.152594 \\ \mathrm{C} & 3.408580 & 1.594781 & 0.972316 \\ \mathrm{H} & 1.796153 & 2.208519 & -0.102640 \\ \mathrm{O} & 3.637632 & -1.708671 & -0.876922 \\ \mathrm{O} & 2.202893 & -1.434001 & 0.840273 \\ \mathrm{H} & 3.794924 & -2.560173 & -0.422739 \\ \mathrm{O} & 3.274068 & 2.753992 & 1.646684 \\ \mathrm{O} & 4.340030 & 0.823220 & 1.069945 \\ \mathrm{H} & 4.060611 & 2.841908 & 2.215495 \\ \mathrm{Br} & -0.961174 & -2.419760 & -0.925585\end{array}$


$\mathrm{TS}-1_{A}$

SCF Energy: -1076.44423691

Num. Imaginary Frequencies: 1

$\begin{array}{lrrr}\mathrm{C} & 1.744620 & -1.063109 & -0.350203 \\ \mathrm{C} & 2.660115 & -2.078567 & -0.616508 \\ \mathrm{C} & 2.419677 & -2.860181 & -1.747556 \\ \mathrm{C} & 1.311006 & -2.656502 & -2.601345 \\ \mathrm{C} & 0.399951 & -1.654304 & -2.328627 \\ \mathrm{C} & 0.613639 & -0.846201 & -1.189234 \\ \mathrm{H} & 3.516837 & -2.242979 & 0.022191 \\ \mathrm{H} & 3.122262 & -3.653668 & -1.985443 \\ \mathrm{H} & 1.184704 & -3.289784 & -3.472845 \\ \mathrm{H} & -0.453231 & -1.482757 & -2.977848 \\ \mathrm{C} & -0.151303 & 0.209491 & -0.640522 \\ \mathrm{~N} & 1.724482 & -0.121184 & 0.681283 \\ \mathrm{C} & 0.686012 & 0.822732 & 0.444656 \\ \mathrm{C} & -1.386476 & 0.858607 & -1.234436 \\ \mathrm{H} & 0.189675 & 1.194251 & 1.332025 \\ \mathrm{C} & 2.635694 & -0.066574 & 1.747007 \\ \mathrm{O} & 3.551230 & -0.839395 & 1.888110 \\ \mathrm{O} & 2.318800 & 0.949695 & 2.564625 \\ \mathrm{H} & 2.985742 & 0.965708 & 3.276446 \\ \mathrm{C} & -2.496004 & -0.019018 & -0.618094 \\ \mathrm{H} & -1.372539 & 0.851918 & -2.326787 \\ \mathrm{H} & -1.465109 & 1.896901 & -0.907512 \\ \mathrm{C} & -3.807365 & 0.672396 & -0.252496 \\ \mathrm{H} & -2.783842 & -0.820812 & -1.316926 \\ \mathrm{~N} & -1.752133 & -0.570527 & 0.515484 \\ \mathrm{C} & -2.158712 & -1.498976 & 1.319183 \\ \mathrm{O} & -3.994798 & 1.829695 & -0.873940 \\ \mathrm{O} & -4.623828 & 0.174733 & 0.509189 \\ \mathrm{H} & -4.873807 & 2.182774 & -0.624395 \\ \mathrm{O} & -1.248976 & -2.101920 & 2.073835 \\ \mathrm{O} & -3.391720 & -1.935840 & 1.495875 \\ \mathrm{H} & -1.702446 & -2.698893 & 2.697503 \\ \mathrm{Br} & 1.433693 & 2.562080 & -0.459392 \\ \mathrm{H} & -4.044743 & -1.267976 & 1.132435\end{array}$


TS $-2_{A}$

SCF Energy: -1076.44042578

Num. Imaginary Frequencies: 1

$\begin{array}{lrrr}\mathrm{C} & -1.749129 & -0.799166 & 0.770221 \\ \mathrm{C} & -2.587570 & -1.627534 & 1.498169 \\ \mathrm{C} & -2.462205 & -1.573162 & 2.893820 \\ \mathrm{C} & -1.540516 & -0.729257 & 3.542741 \\ \mathrm{C} & -0.708049 & 0.098977 & 2.804675 \\ \mathrm{C} & -0.810099 & 0.062736 & 1.400043 \\ \mathrm{H} & -3.297474 & -2.284591 & 1.014728 \\ \mathrm{H} & -3.105729 & -2.208739 & 3.494842 \\ \mathrm{H} & -1.490521 & -0.725274 & 4.626288 \\ \mathrm{H} & -0.007218 & 0.768250 & 3.293787 \\ \mathrm{C} & -0.090257 & 0.751855 & 0.384894 \\ \mathrm{~N} & -1.619641 & -0.632447 & -0.631757 \\ \mathrm{C} & -0.682718 & 0.326531 & -0.890372 \\ \mathrm{C} & 1.248530 & 1.410546 & 0.490321 \\ \mathrm{H} & -0.230323 & 0.412920 & -1.864684 \\ \mathrm{C} & -2.377930 & -1.325768 & -1.612060 \\ \mathrm{O} & -3.184836 & -2.173365 & -1.336112 \\ \mathrm{O} & -2.044995 & -0.887220 & -2.829405 \\ \mathrm{H} & -2.576267 & -1.384365 & -3.480266 \\ \mathrm{C} & 2.296660 & 0.261409 & 0.464628 \\ \mathrm{H} & 1.330753 & 2.006187 & 1.402692 \\ \mathrm{H} & 1.409955 & 2.072762 & -0.361535 \\ \mathrm{C} & 3.731408 & 0.767145 & 0.262123 \\ \mathrm{H} & 2.311271 & -0.225310 & 1.454152 \\ \mathrm{~N} & 1.844179 & -0.669633 & -0.560107 \\ \mathrm{C} & 2.378072 & -1.829133 & -0.728567 \\ \mathrm{O} & 3.853191 & 2.091831 & 0.236112 \\ \mathrm{O} & 4.683344 & 0.009134 & 0.159037 \\ \mathrm{H} & 4.800623 & 2.312518 & 0.122766 \\ \mathrm{O} & 1.723166 & -2.702309 & -1.497466 \\ \mathrm{O} & 3.506185 & -2.325594 & -0.229836 \\ \mathrm{H} & 2.291064 & -3.483211 & -1.626686 \\ \mathrm{Br} & -1.579421 & 2.473258 & -0.671048 \\ \mathrm{H} & 4.106223 & -1.581516 & 0.051201\end{array}$




\section{General synthetic methods.}

Solvents were dried according to published methods and distilled before use. All reagents were commercial compounds of the highest purity available. Reactions were carried out under an argon atmosphere, unless indicated otherwise. Analytical TLC was performed on aluminium plates with Merck Kieselgel $60 \mathrm{~F}_{254}$ and visualised by UV irradiation $(254 \mathrm{~nm})$ or by staining with a methanolic solution of phosphomolibdic acid. Flash-column chromatography was carried out using Merck Kieselgel 60 (230-400 mesh) under pressure. IR spectra were obtained on a JASCO IR 4200 spectrophotometer from a thin film deposited onto $\mathrm{NaCl}$ glass. Specific rotations were obtained on a JASCO P-1020 polarimeter. Mass spectra and HRMS (ESI ${ }^{+}$) were taken on a Apex III FT ICR MS (Bruker Daltonics) and $\left(\mathrm{FAB}^{+}\right)$on a Micromass AutoSpec apparatus. ${ }^{1} \mathrm{H}-\mathrm{NMR}$ spectra were recorded in $\mathrm{CDCl}_{3}$ and $\left(\mathrm{CD}_{3}\right)_{2} \mathrm{CO}$ at ambient temperature on a Bruker AMX-400 spectrometer at $400 \mathrm{MHz}$ with residual protic solvent as the internal reference $\left(\mathrm{CDCl}_{3}, \delta_{\mathrm{H}}=7.24 \mathrm{ppm} ;\left(\mathrm{CD}_{3}\right)_{2} \mathrm{CO}, \delta_{\mathrm{H}}=2.05 \mathrm{ppm}\right)$; chemical shifts $(\delta)$ are given in parts per million (ppm) and coupling constants $(J)$ are given in Hertz $(\mathrm{Hz})$. The proton spectra are reported as follows: $\delta$ (multiplicity, coupling constant $J$, number of protons, assignment). ${ }^{13} \mathrm{C}$-NMR spectra were recorded in $\mathrm{CDCl}_{3}$ at ambient temperature on the same spectrometer at $100 \mathrm{MHz}$, with the central peak of $\mathrm{CDCl}_{3}\left(\delta_{\mathrm{C}}=77.23 \mathrm{ppm}\right)$ or as the internal reference. DEPT135 sequences were used to aid in the assignment of signals in the ${ }^{13} \mathrm{C}-\mathrm{NMR}$ spectra. HPLC analyses were performed on a Waters 510 apparatus with a differential refractometer detector Waters 410.

\subsection{Synthesis of the open-chain precursors}

Some of the precursors of the 3a-bromohexahydropirrolo[2,3-b]indoles have been already described and characterized elsewhere $\left(\mathbf{3 a},{ }^{1} \mathbf{3} \mathbf{b},{ }^{2} \mathbf{3 f}^{3}\right)$.

(R) 3-(2-Methoxycarbonyl-2-methoxycarbonylamino-ethyl)-indole-1-carboxylic acid tert-butyl ester (3c)<smiles>COC(=O)N[C@@H](Cc1cn(C(=O)OC(C)(C)C)c2ccccc12)C(=O)OC</smiles>

\footnotetext{
${ }^{1}$ Depew, M. K.; Marsden, S. P.; Zatorska, D.; Zatorski, A.; Bornmann W. G.; Danishefsky, S. J. J. Am. Chem. Soc. 1999, 121, 11953.

${ }^{2}$ Hewitt, P. R.; Cleator, E.; Ley, S. V. Org. Biomol. Chem. 2004, 2417.

${ }^{3}$ Crich, D.; Huang, X. J. Org. Chem. 1999, 64, 7218.
} 
To a solution of $\mathrm{N}_{\alpha}$-methoxycarbonyl-D-tryptophan methyl ester ${ }^{4}$ (304 mg, $1.1 \mathrm{mmol}$ ) in dichloromethane $(11 \mathrm{~mL})$ were added $\mathrm{NaOH}(130 \mathrm{mg}, 3.4 \mathrm{mmol}, 3.0$ equiv) and $\mathrm{Bu}_{4} \mathrm{NHSO}_{4}\left(40 \mathrm{mg}, 0.1 \mathrm{mmol}, 10 \mathrm{~mol} \%\right.$ ). The mixture was stirred for $30 \mathrm{~min}$ at $25^{\circ} \mathrm{C}$, then $(\mathrm{BOC})_{2} \mathrm{O}(370 \mathrm{mg}, 1.7 \mathrm{mmol}, 1.5$ equiv) was added and stirring was continued for $20 \mathrm{~h}$. The reaction mixture was filtered through a short path of Celite ${ }^{\circledR}$ and the solvent was removed under reduced pressure. The crude material was purified by flash chromatography (70:30, hexanes/EtOAc) to afford $368 \mathrm{mg}(89 \%$ yield) of the title compound as a colourless oil.

${ }^{1} \mathbf{H}$ NMR $\left(400 \mathrm{MHz}, \mathrm{CDCl}_{3}\right) \delta 8.1-7.9(\mathrm{~m}, 1 \mathrm{H}, \mathrm{ArH}), 7.46(\mathrm{~d}, J=7.7 \mathrm{~Hz}, 1 \mathrm{H}, \mathrm{ArH})$, 7.39 (s, 1H, ArH), 7.29 (t, J=7.3 Hz, 1H, ArH), 7.20 (t, J = 7.1 Hz, 1H, ArH), 5.44 (d, $J=8.1 \mathrm{~Hz}, 1 \mathrm{H}, \mathrm{NH}), 4.8-4.7(\mathrm{~m}, 1 \mathrm{H}, \mathrm{H} 2), 3.65\left(\mathrm{~s}, 3 \mathrm{H}, \mathrm{CO}_{2} \mathrm{CH}_{3}\right), 3.63(\mathrm{~s}, 3 \mathrm{H}$, $\left.\mathrm{CO}_{2} \mathrm{CH}_{3}\right), 3.2-3.1(\mathrm{~m}, 2 \mathrm{H}, \mathrm{H} 3 \mathrm{~A} / \mathrm{H} 3 \mathrm{~B}), 1.63\left(\mathrm{~s}, 9 \mathrm{H}, \mathrm{C}\left(\mathrm{CH}_{3}\right)_{3}\right) \mathrm{ppm}$.

${ }^{13}$ C NMR (100 MHz, $\left.\mathrm{CDCl}_{3}\right) \delta 172.2\left(\mathrm{~s}, \underline{\mathrm{CO}}_{2} \mathrm{CH}_{3}\right), 156.5$ (s, OCON), 149.6 (s), 135.3 (s), 130.5 (d), 124.6 (d), 122.6 (d), 118.8 (d), 115.3 (d), 115.0 (s), 83.7(s, $\left.\underline{\mathrm{C}}\left(\mathrm{CH}_{3}\right)_{3}\right), 54.1$ (d), $52.4\left(\mathrm{q}, \mathrm{OCH}_{3}\right), 52.3\left(\mathrm{q}, \mathrm{OCH}_{3}\right), 28.2\left(\mathrm{q}, 3 \mathrm{x}, \mathrm{C}\left(\underline{\mathrm{CH}_{3}}\right)_{3}\right), 27.8(\mathrm{t}) \mathrm{ppm}$.

IR (NaCl) v 3357 (s, N-H), 3006 (s, C-H), 2979 (s, C-H), 2953 (s, C-H), 1731 (s, CO), 1519 (s), 1372 (m), 1257 (s), 1157 (s), 1087 (s), 1172 (s), 750 (s) cm $\mathrm{cm}^{-1}$.

MS $\left(\mathrm{ESI}^{+}\right) \mathrm{m} / \mathrm{z}(\%) 399\left([\mathrm{M}+\mathrm{Na}]^{+}, 100\right), 343$ (5), 291 (10).

HRMS (ESI ${ }^{+}$) calcd for $\mathrm{C}_{19} \mathrm{H}_{24} \mathrm{~N}_{2} \mathrm{NaO}_{6}, 399.1526$; found 399.1520 .

$[\alpha]_{\mathrm{D}}^{26}-41^{\mathrm{o}}\left(c 0.25, \mathrm{CHCl}_{3}\right)$.

\footnotetext{
${ }^{4}$ Zembower, D. E.; Gilbert, J. A.; Ames, M. M. J. Med. Chem. 1993, 36, 305
} 

acid methyl ester (3d)

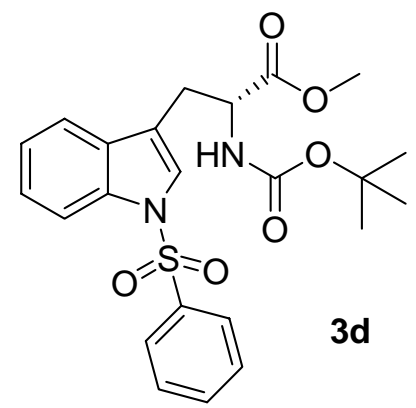

Benzenesulphonyl chloride ( $125 \mu \mathrm{L}, 0.98 \mathrm{mmol}, 1.2$ equiv) was added dropwise to a suspension of $\mathrm{N}_{\alpha}$-t-butyloxycarbonyl-D-tryptophan methyl ester ${ }^{5}$ (261 $\mathrm{mg}, 0.82 \mathrm{mmol}$ ), $\mathrm{NaOH}$ (99 mg, $2.46 \mathrm{mmol}, 3.0$ equiv) and $\mathrm{Bu}_{4} \mathrm{NHSO}_{4}$ (14 mg, $0.04 \mathrm{mmol}, 5 \mathrm{~mol} \%$ ) in dichloromethane $(5 \mathrm{~mL})$. The resulting mixture was stirred for $3 \mathrm{~h}$ at $25^{\circ} \mathrm{C}$. The reaction was worked up by the addition of a saturated $\mathrm{NH}_{4} \mathrm{Cl}$ aqueous solution $(10 \mathrm{~mL})$ and EtOAc $(10 \mathrm{~mL})$ and then extracted with EtOAc $(3 \times 15 \mathrm{~mL})$. The combined organic layers were dried over $\mathrm{Na}_{2} \mathrm{SO}_{4}$ and the solvents were removed under reduced pressure. The residue was purified by flash chromatography (75:25, hexanes/EtOAc) to afford $335 \mathrm{mg}$ ( $89 \%$ yield) of the title compound as a white foam.

${ }^{1} \mathbf{H}$ NMR $\left(400 \mathrm{MHz}, \mathrm{CDCl}_{3}\right) \delta 7.95(\mathrm{~d}, J=8.3 \mathrm{~Hz}, 1 \mathrm{H}, \mathrm{ArH}), 7.82(\mathrm{~d}, J=7.5 \mathrm{~Hz}, 2 \mathrm{H}$, ArH), 7.5-7.4 (m, 1H, ArH) 7.4-7.3 (m, 3H, ArH), 7.3-7.2 (m, 2H, ArH), 7.21 (t, $J=7.5$ $\mathrm{Hz}, 1 \mathrm{H}, \mathrm{ArH}), 5.03$ (d, $J=7.7 \mathrm{~Hz}, 1 \mathrm{H}, \mathrm{NH}), 4.61$ (dd, $J=13.3,5.7 \mathrm{~Hz}, 1 \mathrm{H}, \mathrm{H} 2), 3.59$ (s, 3H, $\left.\mathrm{CO}_{2} \mathrm{CH}_{3}\right), 3.2-3.0(\mathrm{~m}, 2 \mathrm{H}, \mathrm{H} 3 \mathrm{~A} / \mathrm{H} 3 \mathrm{~B}), 1.35$ (s, 9H, $\left.\mathrm{CO}_{2} t-\mathrm{Bu}\right) \mathrm{ppm}$.

${ }^{13} \mathrm{C}$ NMR $\left(100 \mathrm{MHz}, \mathrm{CDCl}_{3}\right) \delta 172.2\left(\mathrm{~s}, \underline{\mathrm{CO}}_{2} \mathrm{CH}_{3}\right), 155.2$ (s, OCON), 138.3 (s), 135.3 (s), 134.0 (d), 131.1 (s), 129.5 (d), 126.9 (d, 2x), 125.2(d), 124.6 (d), 123.5 (d), 119.7 (d), 117.7 (s), 113.9 (d), 80.4(s, $\left.\underline{\mathrm{C}}\left(\mathrm{CH}_{3}\right)_{3}\right), 53.4$ (d), 52.6 (q, $\left.\mathrm{CO}_{2} \mathrm{CH}_{3}\right), 28.5$ (q, 3x, $\left.\mathrm{C}\left(\mathrm{CH}_{3}\right)_{3}\right), 28.0$ (t) ppm.

IR (NaCl) v 3393 (br, N-H), 2977 (m, C-H), 2954 (w, C-H), 1743 (s, CO), 1711 (s, CO), 1503 (m), 1447 (s), 1367 (s), 1175 (s), 1023 (m), 749 (s) cm².

MS $\left(\mathrm{ESI}^{+}\right) \mathrm{m} / \mathrm{z}(\%) 481\left([\mathrm{M}+\mathrm{Na}]^{+}, 100\right), 359(45), 212(7)$.

HRMS $\left(\right.$ ESI $\left.^{+}\right)$calcd for $\mathrm{C}_{23} \mathrm{H}_{26} \mathrm{~N}_{2} \mathrm{NaO}_{6} \mathrm{~S}, 481.1403$; found 481.1398 .

$[\alpha]_{\mathrm{D}}^{27}-26^{\mathrm{o}}\left(c 0.23, \mathrm{CHCl}_{3}\right)$.

\footnotetext{
${ }^{5}$ Knudsen, K. R.; Bachmann, S.; Jorgensen, K. A. Chem. Commun. 2003, 2602.
} 
(R)-3-(1-Benzenesulfonyl-1H-indol-3-yl)-2-benzyloxycarbonylamino-propionic acid methyl ester (3e).

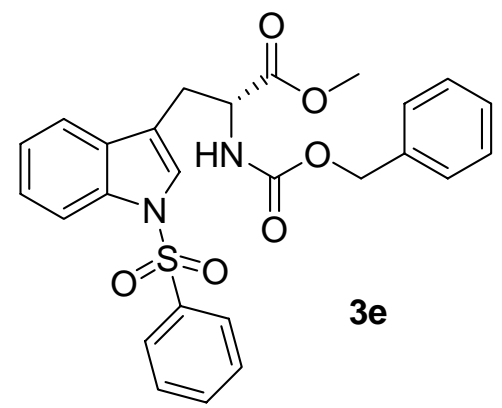

Following the same procedure described for $\mathbf{3 d}$, the tryptophan derivative $3 \mathbf{e}$ was prepared in $92 \%$ yield starting from the $N$-phenylmethoxycarbonyl- $D$-tryptophan methyl ester. $^{2}$

${ }^{1} \mathbf{H}$ NMR $\left(400 \mathrm{MHz}, \mathrm{CDCl}_{3}\right) \delta 7.96(\mathrm{~d}, J=8.3 \mathrm{~Hz}, 1 \mathrm{H}, \mathrm{ArH}), 7.79(\mathrm{~d}, J=7.6 \mathrm{~Hz}, 2 \mathrm{H}$, ArH), 7.5-7.2 (m, 11H, ArH) 7.17 (t, $J=7.5 \mathrm{~Hz}, 1 \mathrm{H}, \mathrm{ArH}), 5.33$ (d, $J=7.7 \mathrm{~Hz}, 1 \mathrm{H}$, $\mathrm{NH}), 5.2-4.9\left(\mathrm{~m}, 2 \mathrm{H}, \underline{\mathrm{C}}_{2} \mathrm{Ph}\right), 4.69$ (dd, $\left.J=13.3,5.6 \mathrm{~Hz}, 1 \mathrm{H}, \mathrm{H} 2\right), 3.60$ (s, 3H, $\mathrm{OCH}_{3}$ ), 3.3-3.1 (m, 2H, H3A/H3B) ppm.

${ }^{13} \mathrm{C}$ NMR $\left(100 \mathrm{MHz}, \mathrm{CDCl}_{3}\right) \delta 171.8\left(\mathrm{~s}, \underline{\mathrm{CO}}_{2} \mathrm{CH}_{3}\right), 155.8(\mathrm{~s}, \mathrm{OCON}), 138.2(\mathrm{~s}), 136.3$ (s), 135.3 (s), 134.0 (d), 130.9, 129.4 (d, 2x), 128.7 (d, 2x), 128.4 (d), 128.3 (d, 2x), 126.8 (d, 2x), 125.2 (d), 124.6 (d), 123.6 (d), 119.6 (d), 117.5, 113.9 (d), 67.2(t, $\left.\mathrm{CH}_{2} \mathrm{Ph}\right), 54.1(\mathrm{~d}), 52.6\left(\mathrm{q}, \mathrm{OCH}_{3}\right), 28.0(\mathrm{t}) \mathrm{ppm}$.

IR (NaCl) v 3383 (br, N-H), $3064(\mathrm{~m}, \mathrm{C}-\mathrm{H}), 3031$ (m, C-H), 2952 (s, C-H), 1722 (s, CO), 1515 (s), 1447 (s), 1366 (s), 1175 (s), 747 (s) $\mathrm{cm}^{-1}$.

MS $\left(\mathrm{ESI}^{+}\right) \mathrm{m} / \mathrm{z}(\%) 515\left([\mathrm{M}+\mathrm{Na}]^{+}, 100\right), 449(98), 432(10)$.

HRMS (ESI ${ }^{+}$) calcd for $\mathrm{C}_{26} \mathrm{H}_{24} \mathrm{~N}_{2} \mathrm{NaO}_{6} \mathrm{~S}, 515.1247$; found 515.1238.

$[\alpha]_{\mathrm{D}}^{25}-44\left(c 0.30, \mathrm{CHCl}_{3}\right)$. 


\subsection{Synthesis of 3a-bromo-hexahydropyrrolo[2,3-b]indoles.}

\section{(2R,3aS,8aS)-3a-Bromo-2,3,3a,8a-tetrahydro-pyrrolo[2,3-b]indole-1,2,8-}

\section{tricarboxylic acid 1,8-di-tert-butyl ester 2-methyl ester (6a)}
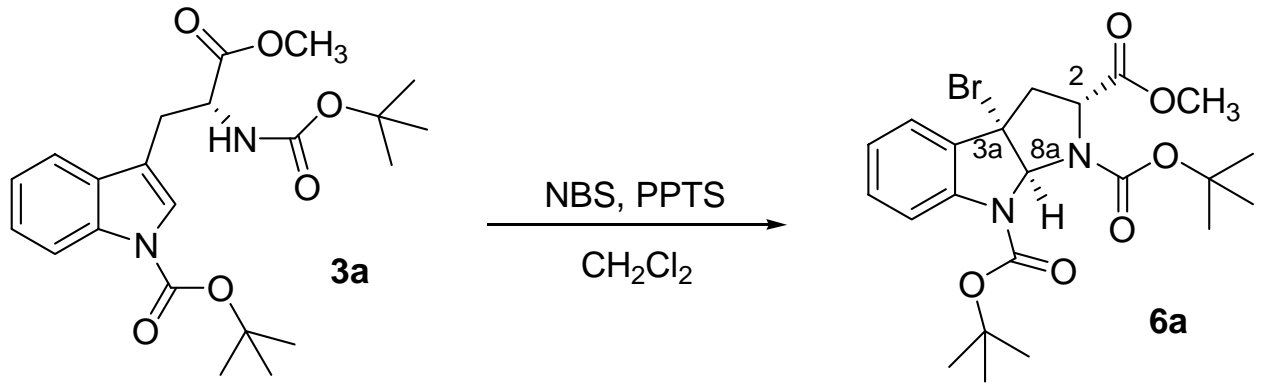

To a solution of the tryptophan derivative 3a (1.633 g, $3.90 \mathrm{mmol})$ in $\mathrm{CH}_{2} \mathrm{Cl}_{2}(33 \mathrm{~mL})$ under argon was added $\mathrm{N}$-bromosuccinimide (695 $\mathrm{mg}, 3.90 \mathrm{mmol}, 1$ equiv) and pyridinium $p$-toluenesulfonate (980 $\mathrm{mg}, 3.90 \mathrm{mmol}, 1$ equiv). The resulting yellow solution was stirred at $25^{\circ} \mathrm{C}$ for 5 hours. The mixture was treated with a $10 \% \mathrm{NaHCO}_{3}$ aqueous solution $(20 \mathrm{~mL})$ and a $10 \% \mathrm{Na}_{2} \mathrm{~S}_{2} \mathrm{O}_{4}$ aqueous solution $(20 \mathrm{~mL})$. The organic layer was dried over $\mathrm{Na}_{2} \mathrm{SO}_{4}$ and the solvents were removed under reduced pressure. The residue was purified by flash chromatography (70:30, hexanes/EtOAc) to afford $1.648 \mathrm{~g}$ (85\%yield) of the title compound as a white foam.

${ }^{1}$ H NMR $\left(400 \mathrm{MHz}, \mathrm{CDCl}_{3}\right) \delta$ 7.7-7.3 (br, 1H, ArH), 7.3-7.2 (m, 2H, ArH), $7.04(\mathrm{t}, J=$ $7.4 \mathrm{~Hz}, 1 \mathrm{H}, \mathrm{ArH}), 6.32$ (s, 1H, H8a), 3.81 (dd, $J=10.2,6.3 \mathrm{~Hz}, 1 \mathrm{H}, \mathrm{H} 2), 3.66$ (s, 3H, $\mathrm{CO}_{2} \mathrm{CH}_{3}$ ), 3.13 (dd, $J=12.6,6.3 \mathrm{~Hz}, 1 \mathrm{H}, \mathrm{H} 3 \mathrm{~A}$ ), 2.74 (dd, $J=12.6,10.2 \mathrm{~Hz}, 1 \mathrm{H}, \mathrm{H} 3 \mathrm{~B}$ ), $1.55\left(\mathrm{~s}, 9 \mathrm{H}, \mathrm{CO}_{2} t-\mathrm{Bu}\right), 1.4-1.2\left(\mathrm{br}, 9 \mathrm{H}, \mathrm{CO}_{2} t-\mathrm{Bu}\right) \mathrm{ppm}$.

${ }^{13}$ C NMR (100 MHz, $\left.\mathrm{CDCl}_{3}\right) \delta 171.7\left(\mathrm{~s}, \underline{\mathrm{CO}}_{2} \mathrm{CH}_{3}\right), 152.3$ (s, 2xOCON), 141.7 (s),

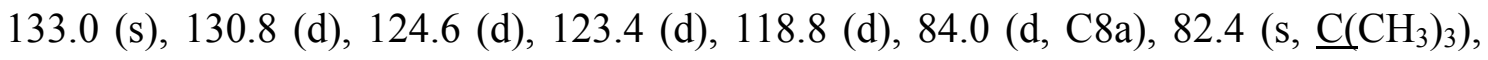

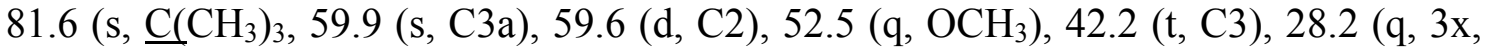
$\left.\mathrm{C}\left(\underline{\mathrm{CH}}_{3}\right)_{3}\right), 28.2\left(\mathrm{q}, \mathrm{C}\left(\underline{\mathrm{C}} \mathrm{H}_{3}\right)_{3}\right) \mathrm{ppm}$.

IR (NaCl) v $2978(\mathrm{w}, \mathrm{C}-\mathrm{H}), 2932(\mathrm{w}, \mathrm{C}-\mathrm{H}), 1752$ (m, CO), 1715 (s, CO), 1604 (w), 1477 (m), 1329 (m), 1254 (m), 1153 (s), 848 (m), 749 (s) $\mathrm{cm}^{-1}$.

MS $\left(\mathrm{ESI}^{+}\right) \mathrm{m} / \mathrm{z}(\%) 521\left([\mathrm{M}+\mathrm{Na}]^{+}\left[{ }^{81} \mathrm{Br}\right], 71\right), 519\left([\mathrm{M}+\mathrm{Na}]^{+}\left[{ }^{79} \mathrm{Br}\right], 100\right), 387(10), 385$ (10), 339 (9), $283(8)$.

HRMS (ESI ${ }^{+}$) calcd for $\mathrm{C}_{22} \mathrm{H}_{29}{ }^{81} \mathrm{BrN}_{2} \mathrm{NaO}_{6}, \quad 521.1080$ and $\mathrm{C}_{22} \mathrm{H}_{29}{ }^{79} \mathrm{BrN}_{2} \mathrm{NaO}_{6}$, 519.1101; found 521.1084 and 519.1102 . 
$[\alpha]_{\mathrm{D}}^{27}+151^{\circ}\left(c 0.39, \mathrm{CHCl}_{3}\right)$

(2R,3aS,8aS)-3a-Bromo-2,3,3a,8a-tetrahydro-pyrrolo[2,3-b]indole-1,2,8tricarboxylic acid 1-benzyl ester 8-tert-butyl ester 2-methyl ester (6b).
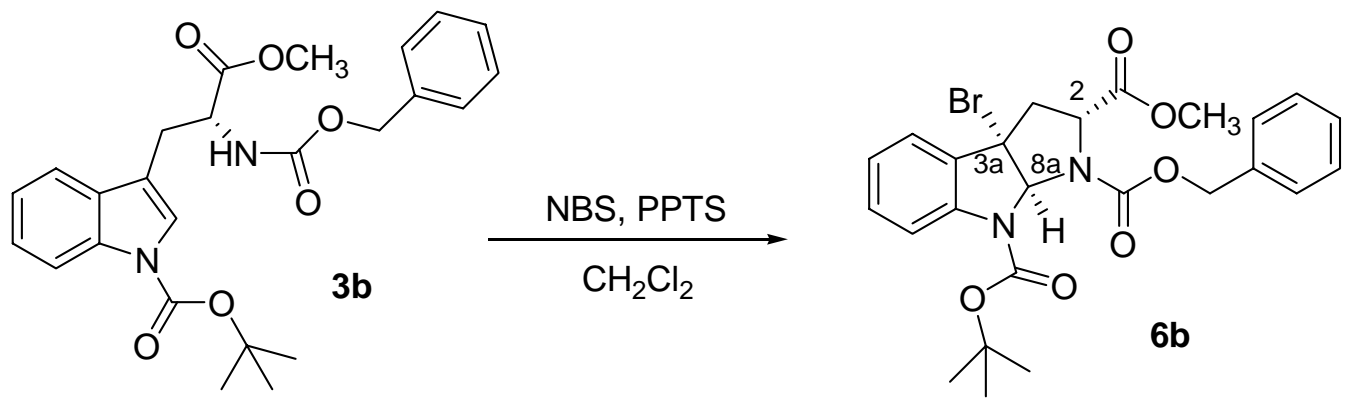

To a solution of the tryptophan derivative $3 \mathbf{b}(1.233 \mathrm{~g}, 2.70 \mathrm{mmol})$ in $\mathrm{CH}_{2} \mathrm{Cl}_{2}(23 \mathrm{~mL})$ under argon was added $\mathrm{N}$-bromosuccinimide (481 $\mathrm{mg}, 2.70 \mathrm{mmol}, 1$ equiv) and pyridinium $p$-toluenesulfonate $(678 \mathrm{mg}, 2.70 \mathrm{mmol}, 1$ equiv). The resulting yellow solution was stirred at $25^{\circ} \mathrm{C}$ for 8 hours. The mixture was treated with a $10 \% \mathrm{NaHCO}_{3}$ aqueous solution $(20 \mathrm{~mL})$ and a $10 \% \mathrm{Na}_{2} \mathrm{~S}_{2} \mathrm{O}_{4}$ aqueous solution $(20 \mathrm{~mL})$. The organic layer was dried over $\mathrm{Na}_{2} \mathrm{SO}_{4}$ and the solvents were removed under reduced pressure. The residue was purified by flash chromatography (75:25, hexanes/EtOAc) to afford $1.248 \mathrm{~g}$ (87\%yield) of the title compound as a colourless oil.

${ }^{1} \mathbf{H}$ NMR $\left(400 \mathrm{MHz}, \mathrm{CDCl}_{3}\right) \delta$ 7.8-7.4 (br , 1H, ArH), 7.5-7.4 (m, 7H, ArH), 7.27.1(m, 1H, ArH), 6.41 (s, 1H, H8a), 5.3-4.7 (br , 2H, $\left.\underline{\mathrm{C}}_{2} \mathrm{Ph}\right), 3.96$ (dd, $J=10.2,6.6$ $\mathrm{Hz}, 1 \mathrm{H}, \mathrm{H} 2), 3.8-3.3\left(\mathrm{~m}, 3 \mathrm{H}, \mathrm{CO}_{2} \mathrm{C}_{3}\right), 3.24$ (dd, $\left.J=12.8,6.6 \mathrm{~Hz}, 1 \mathrm{H}, \mathrm{H} 3 \mathrm{~A}\right), 2.83$ (dd, $J=12.8,10.2 \mathrm{~Hz}, 1 \mathrm{H}, \mathrm{H} 3 \mathrm{~B}), 1.50$ (br , 9H, $\mathrm{CO}_{2}{ }^{t} \mathrm{Bu}$ ) ppm.

${ }^{13} \mathrm{C}$ NMR (100 MHz, $\left.\mathrm{CDCl}_{3}\right) \delta 171.2\left(\mathrm{~s}, \underline{\mathrm{CO}}_{2} \mathrm{Me}\right), 152.2$ (s, 2x, OCOON), 141.5 (s), 136.1 (s), 132.7 (s), 131.1 (d, 2x), 128.6 (d, 2x), 128.3 (d), 124.8 (d, 2x), 123.4 (d), 118.8 (d), 84.1 (d, C8a), 82.6 (s, $\left.\underline{\mathrm{C}}\left(\mathrm{CH}_{3}\right)_{3}\right), 67.6$ (t, $\left.\underline{\mathrm{CH}}_{2} \mathrm{Ph}\right), 59.9$ (C3a), 59.7 (d, C2), $52.7\left(\mathrm{q}, \mathrm{CO}_{2} \underline{\mathrm{CH}}_{3}\right), 41.9$ (t, $\left.\mathrm{C} 3\right), 28.4\left(\mathrm{q}, 3 \mathrm{x}, \mathrm{C}\left(\underline{\mathrm{CH}}_{3}\right)_{3}\right) \mathrm{ppm}$.

IR (NaCl) v $2924(\mathrm{w}, \mathrm{C}-\mathrm{H}), 2854(\mathrm{w}, \mathrm{C}-\mathrm{H}), 1714$ (m, CO), 1605 (s, CO), 1504 (m), 1450 (m), 1235 (m), $1116(\mathrm{w}), 929(\mathrm{w}) \mathrm{cm}^{-1}$. 
MS $\left(\mathrm{FAB}^{+}\right) \mathrm{m} / \mathrm{z}(\%) 533\left([\mathrm{M}+1]^{+}\left[{ }^{81} \mathrm{Br}\right], 15\right), 531\left([\mathrm{M}+1]^{+}\left[{ }^{79} \mathrm{Br}\right], 16\right), 477(53), 475$

(53), 433 (21), 432 (31), 431 (24), 430 (28), 351 (66), 307 (100), 217 (40).

HRMS $\left(\mathrm{FAB}^{+}\right)$calcd for $\mathrm{C}_{25} \mathrm{H}_{28}{ }^{79} \mathrm{BrN}_{2} \mathrm{O}_{6} \mathrm{~S}, 531.1131$; found 531.1133.

$[\alpha]_{\mathrm{D}}^{23}+157^{\circ}\left(c 0.28, \mathrm{CHCl}_{3}\right)$.

(2R,3aS,8aS)-3a-Bromo-2,3,3a,8a-tetrahydro-pyrrolo[2,3-b]indole-1,2,8-

tricarboxylic acid 8-tert-butyl ester 1,2-dimethyl ester (6c).
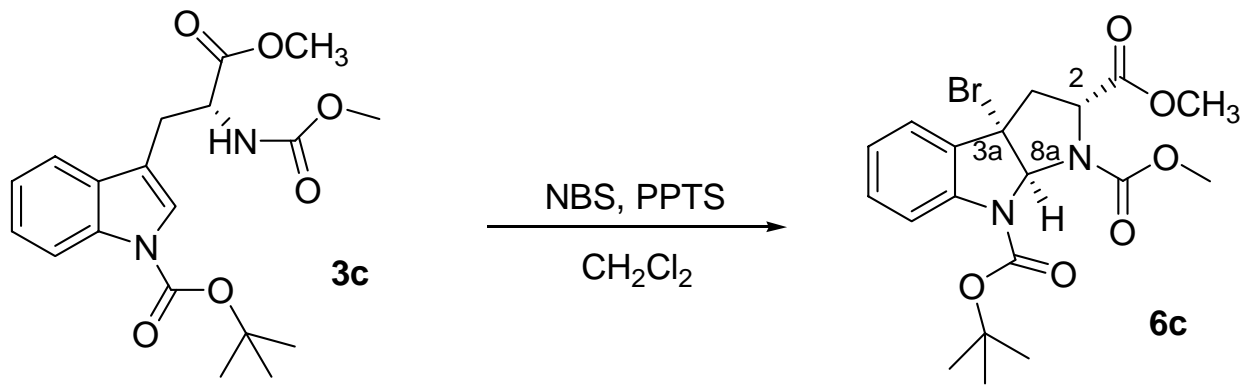

To a solution of the tryptophan derivative $3 \mathrm{c}(185 \mathrm{mg}, 0.49 \mathrm{mmol})$ in $\mathrm{CH}_{2} \mathrm{Cl}_{2}(4 \mathrm{~mL})$ under argon was added $N$-bromosuccinimide ( $88 \mathrm{mg}, 0.49 \mathrm{mmol}, 1$ equiv) and pyridinium $p$-toluenesulfonate (123 mg, $0.49 \mathrm{mmol}, 1$ equiv). The resulting yellow solution was stirred at $25{ }^{\circ} \mathrm{C}$ for 4 hours. The mixture was treated with a $10 \% \mathrm{NaHCO}_{3}$ aqueous solution $(5 \mathrm{~mL})$ and a $10 \% \mathrm{Na}_{2} \mathrm{~S}_{2} \mathrm{O}_{4}$ aqueous solution $(5 \mathrm{~mL})$. The organic layer was dried over $\mathrm{Na}_{2} \mathrm{SO}_{4}$ and the solvents were removed under reduced pressure. The residue was purified by flash chromatography (70:30, hexanes/EtOAc) to afford $201 \mathrm{mg}$ (90\%yield) of the title compound as a colourless oil.

${ }^{1}$ H NMR $\left(400 \mathrm{MHz}, \mathrm{CDCl}_{3}\right) \delta$ 7.6-7.5 (br, 1H, ArH), 7.4-7.2 (m, 2H, ArH), 7.10 (t, $J=$ $7.5 \mathrm{~Hz}, 1 \mathrm{H}, \mathrm{ArH}), 6.34$ (s, 1H, H8a), 3.92 (dd, $J=10.3,6.5 \mathrm{~Hz}, 1 \mathrm{H}, \mathrm{H} 2), 3.73$ (s, 3H, $\left.\mathrm{OCH}_{3}\right), 3.23(\mathrm{dd}, J=12.7,6.4 \mathrm{~Hz}, 1 \mathrm{H}, \mathrm{H} 3 \mathrm{~A}), 2.82$ (dd, $\left.J=12.7,10.3 \mathrm{~Hz}, 1 \mathrm{H}, \mathrm{H} 3 \mathrm{~B}\right)$, $1.55\left(\mathrm{~s}, 9 \mathrm{H}, \mathrm{CO}_{2} t-\mathrm{Bu}\right) \mathrm{ppm}$.

${ }^{13} \mathrm{C}$ NMR (100 MHz, $\left.\mathrm{CDCl}_{3}\right) \delta 171.1\left(\mathrm{~s}, \mathrm{CO}_{2} \mathrm{CH}_{3}\right), 151.9$ (s, 2xOCON), 141.2 (s), 132.3 (s), 130.8 (d), 123.3 (d), 123.1 (d), 118.2 (d), 83.7 (d, C8a), 82.2 (s, $\left.\underline{\mathrm{CCH}}_{3}\right), 59.7$ $(\mathrm{s}, \mathrm{C} 3 \mathrm{a}), 59.5(\mathrm{~d}, \mathrm{C} 2), 52.9\left(\mathrm{q}, \mathrm{OCH}_{3}\right), 52.6\left(\mathrm{q}, \mathrm{OCH}_{3}\right), 41.8(\mathrm{t}, \mathrm{C} 3), 28.2(\mathrm{q}, 3 \mathrm{x}$, $\left.\mathrm{C}\left(\underline{\mathrm{CH}}_{3}\right)_{3}\right) \mathrm{ppm}$. 
IR (NaCl) $v 2979$ (m, C-H), $2954(\mathrm{~m}, \mathrm{C}-\mathrm{H}), 1752$ (s, CO), 1714 (s, CO), 1604 (w), 1448 (s), 1392 (s), 1334 (s), 1157 (s), 732 (s) cm $\mathrm{cm}^{-1}$.

MS $\left(\mathrm{ESI}^{+}\right) \mathrm{m} / \mathrm{z}(\%) 479\left([\mathrm{M}+\mathrm{Na}]^{+}\left[{ }^{81} \mathrm{Br}\right], 73\right), 477\left([\mathrm{M}+\mathrm{Na}]^{+}\left[{ }^{79} \mathrm{Br}\right], 76\right), 423(16), 421$ (16), 297 (100), 275 (17).

HRMS (ESI ${ }^{+}$) calcd for $\mathrm{C}_{19} \mathrm{H}_{23}{ }^{81} \mathrm{BrN}_{2} \mathrm{NaO}_{6}, 479.0611$ and $\mathrm{C}_{19} \mathrm{H}_{23}{ }^{79} \mathrm{BrN}_{2} \mathrm{NaO}_{6}$, 477.0631; found 479.0604 and 477.0626 .

$[\alpha]_{\mathrm{D}}^{28}+126^{\circ}\left(c 0.70, \mathrm{CHCl}_{3}\right)$.

(2R,3aS,8aS)-8-Benzenesulfonyl-3a-bromo-3,3a,8,8a-tetrahydro-2H-pyrrolo[2,3-

b]indole-1,2-dicarboxylic acid 1-tert-butyl ester 2-methyl ester (6d).
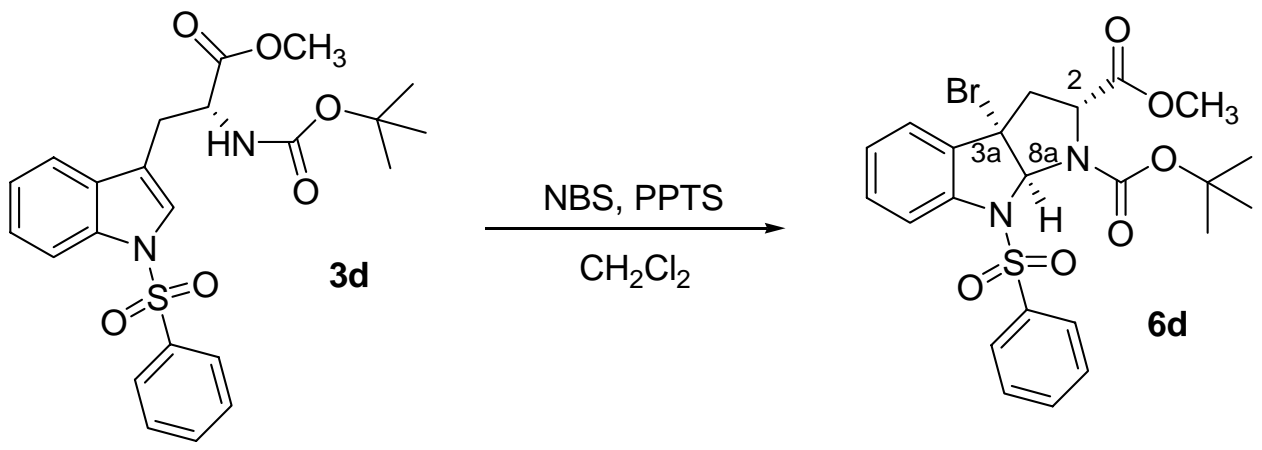

To a solution of the tryptophan derivative $3 \mathbf{d}(103 \mathrm{mg}, 0.22 \mathrm{mmol})$ in $\mathrm{CH}_{2} \mathrm{Cl}_{2}(2 \mathrm{~mL})$ under argon was added $\mathrm{N}$-bromosuccinimide (40 mg, $0.22 \mathrm{mmol}, 1$ equiv) and pyridinium $p$-toluenesulfonate $(57 \mathrm{mg}, 0.22 \mathrm{mmol}, 1$ equiv). The resulting yellow solution was stirred at $25^{\circ} \mathrm{C}$ for 8 hours. The mixture was diluted with $\mathrm{CH}_{2} \mathrm{Cl}_{2}(5 \mathrm{~mL}) \mathrm{a}$ nd treated with a $10 \% \mathrm{NaHCO}_{3}$ aqueous solution $(5 \mathrm{~mL})$ and a $10 \% \mathrm{Na}_{2} \mathrm{~S}_{2} \mathrm{O}_{4}$ aqueous solution $(5 \mathrm{~mL})$. The organic layer was dried over $\mathrm{Na}_{2} \mathrm{SO}_{4}$ and the solvents were removed under reduced pressure. The residue was purified by flash chromatography (70:30, hexanes/EtOAc) to afford $77 \mathrm{mg}$ (65\%yield) of the title compound as a white foam.

${ }^{1}$ H NMR (400 MHz, $\left.\mathrm{CDCl}_{3}\right) \delta$ 7.9-7.6 (m, 2H, ArH), 7.6-7.5 (m, 1H, ArH), $7.43(\mathrm{t}, J=$ $7.4 \mathrm{~Hz}, 1 \mathrm{H}, \mathrm{ArH}), 7.3-7.2$ (m, 4H, ArH), 7.14 (t, $J=7 . \mathrm{Hz}, 1 \mathrm{H}, \mathrm{ArH}), 6.4-6.2(\mathrm{br}, 1 \mathrm{H}$, 
H8a), 3.8-3.7 (m, 1H, H2), $3.70\left(\mathrm{~s}, 1 \mathrm{H}, \mathrm{CO}_{2} \mathrm{CH}_{3}\right), 3.03$ (dd, $\left.J=12.5,5.7 \mathrm{~Hz}, 1 \mathrm{H}, \mathrm{H} 3 \mathrm{~A}\right)$, $2.74(\mathrm{t}, J=12.5 \mathrm{~Hz}, 1 \mathrm{H}, \mathrm{H} 3 \mathrm{~B}), 1.6-1.2\left(\mathrm{br}, 9 \mathrm{H}, \mathrm{CO}_{2} \mathrm{t}-\mathrm{Bu}\right) \mathrm{ppm}$.

${ }^{13} \mathrm{C}$ NMR (100 MHz, $\left.\mathrm{CDCl}_{3}\right) \delta 171.0\left(\mathrm{~s}, \mathrm{CO}_{2} \mathrm{CH}_{3}\right), 153.3$ (s, OCON), 140.5 (s), 138.6 (s), 134.2 (s), 133.6(d), 131.1 (d, 2x), 128.8 (d, 2x), $128.5(\mathrm{~d}), 126.6$ (d), 124.1 (d), 119.0 (d), 86.8(d, C8a), $83.3\left(\mathrm{~s}, \underline{\mathrm{C}}\left(\mathrm{CH}_{3}\right)_{3}\right), 59.6(\mathrm{~d}, \mathrm{C} 2), 59.5(\mathrm{~s}, \mathrm{C} 3 \mathrm{a}), 52.6\left(\mathrm{q}, \mathrm{OCH}_{3}\right), 43.4$ (t, C3), 28.4 (q, 3x, $\left.\mathrm{C}\left(\underline{\mathrm{CH}}_{3}\right)_{3}\right) \mathrm{ppm}$.

IR (NaCl) v 2979 (w, C-H), 2953 (w, C-H), 1752 (s, CO), 1699 (s), 1393 (s), 1367 (s), $1170(\mathrm{~s}), 1031(\mathrm{~m}), 755(\mathrm{~s}) \mathrm{cm}^{-1}$.

MS $\left.\left(\mathrm{ESI}^{+}\right) \mathrm{m} / \mathrm{z}(\%) 561\left([\mathrm{M}+\mathrm{Na}]^{+}\left[{ }^{81} \mathrm{Br}\right], 100\right), 559([\mathrm{M}+\mathrm{Na}]]^{+}\left[{ }^{79} \mathrm{Br}\right], 95\right), 483(48), 481$ (48), 439 (51), 437 (51), 357 (15).

HRMS $\left(\mathrm{ESI}^{+}\right.$) calcd for $\mathrm{C}_{23} \mathrm{H}_{25}{ }^{81} \mathrm{BrN}_{2} \mathrm{NaO}_{6} \mathrm{~S}, 561.0488$ and $\mathrm{C}_{23} \mathrm{H}_{25}{ }^{79} \mathrm{BrN}_{2} \mathrm{NaO}_{6} \mathrm{~S}$, 559.0509; found 561.0481 and 559.0502 .

$[\alpha]_{\mathrm{D}}^{28}+147^{\circ}\left(c 0.15, \mathrm{CHCl}_{3}\right)$.

(2R,3aS,8aS)-8-Benzenesulfonyl-3a-bromo-3,3a,8,8a-tetrahydro-2 $\mathrm{H}$-pyrrolo[2,3-

b]indole-1,2-dicarboxylic acid 1-benzyl ester 2-methyl ester (6e).
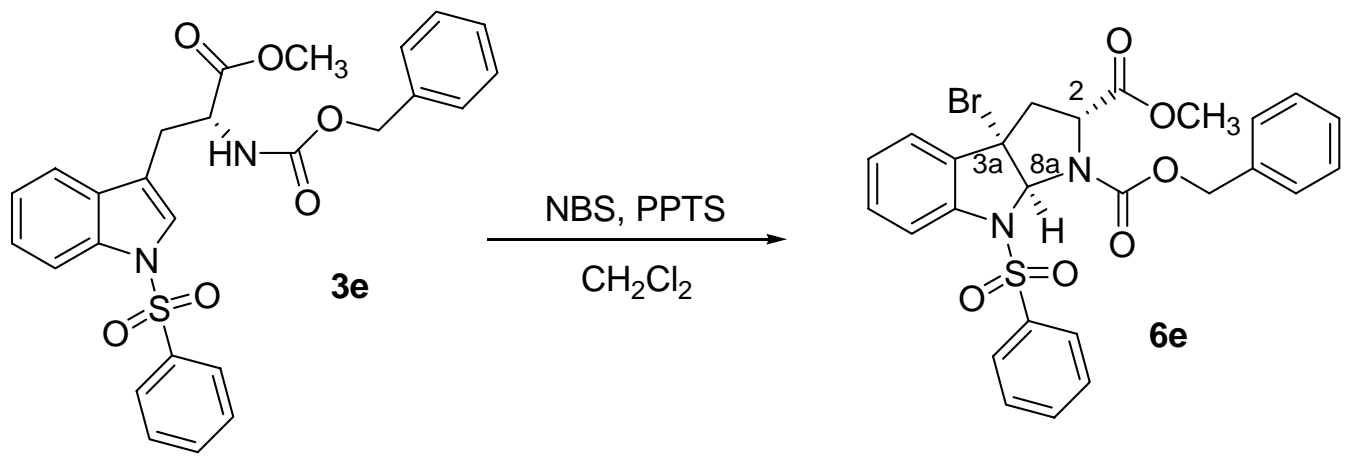

To a solution of the tryptophan derivative $3 \mathbf{e}(207 \mathrm{~g}, 0.42 \mathrm{mmol})$ in $\mathrm{CH}_{2} \mathrm{Cl}_{2}(3.5 \mathrm{~mL})$ under argon was added $\mathrm{N}$-bromosuccinimide (75 $\mathrm{mg}, 0.42 \mathrm{mmol}, 1$ equiv) and pyridinium $p$-toluenesulfonate (106 $\mathrm{mg}, 0.42 \mathrm{mmol}, 1$ equiv). The resulting yellow solution was stirred at $25^{\circ} \mathrm{C}$ for 2 hours. The mixture was diluted with $\mathrm{CH}_{2} \mathrm{Cl}_{2}(7 \mathrm{~mL})$ and treated with a $10 \% \mathrm{NaHCO}_{3}$ aqueous solution $(10 \mathrm{~mL})$ and a $10 \% \mathrm{Na}_{2} \mathrm{~S}_{2} \mathrm{O}_{4}$ aqueous solution $(10 \mathrm{~mL})$. The organic layer was dried over $\mathrm{Na}_{2} \mathrm{SO}_{4}$ and the solvents were 
removed under reduced pressure. The residue was purified by flash chromatography (65:25, hexanes/EtOAc) to afford $173 \mathrm{mg}$ (72\%yield) of the title compound as a colourless oil.

${ }^{1}$ H NMR (400 MHz, $\left.\mathrm{CDCl}_{3}\right) \delta$ 7.9-7.6 (m, 2H, ArH), 7.60 (d, $\left.J=7.6 \mathrm{~Hz}, 1 \mathrm{H}, \mathrm{ArH}\right)$, 7.5-7.4 (m, 2H, ArH) 7.4-7.2 (m, 7H, ArH), 7.3-7.2 (m, 1H, ArH), 7.16 (t, J = 7.5 Hz, 1H, ArH), 6.3-6.2 (br, 1H, H8a), 5.5-4.8 (m, 2H, $\left.\mathrm{OC}_{2} \mathrm{Ph}\right), 3.9-3.8$ (m, 1H, H2), 3.7-3.6 (br, $3 \mathrm{H}, \mathrm{CO}_{2} \underline{\mathrm{CH}}_{3}$ ), 3.07 (dd, $J=12.8,6.1 \mathrm{~Hz}, 1 \mathrm{H}, \mathrm{H} 3 \mathrm{~A}$ ), 2.76 (dd, $J=12.8,10.5 \mathrm{~Hz}$, 1H, H3B) ppm.

${ }^{13}$ C NMR (100 MHz, $\left.\mathrm{CDCl}_{3}\right) \delta 170.6\left(\mathrm{~s}, \underline{\mathrm{CO}}_{2} \mathrm{CH}_{3}\right), 153.8$ (s, OCON), 140.4 (s), 138.5 (s), 136.0 (s), 134.0 (s), 133.7 (d), 131.2 (d), 128.9 (d, 2x), 128.8 (d, 2x), 126.6 (d, 2x), 124.0 (d), 119.1 (d, 2x), 86.8 (s, C3a), 68.1 (t, $\left.\underline{\mathrm{CH}}_{2} \mathrm{Ph}\right), 59.4$ (d, C2), 59.2 (s, C3a), 52.7(q, $\left.\mathrm{OCH}_{3}\right), 43.3(\mathrm{t}, \mathrm{C} 3) \mathrm{ppm}$.

IR (NaCl) v $3065(\mathrm{~m}, \mathrm{C}-\mathrm{H}), 3030(\mathrm{~m}, \mathrm{C}-\mathrm{H}), 2952$ (m, C-H), 1750 (s, CO), 1710 (s, CO), 1600 (w), 1408 (s), 1120 (s), 1367 (s), 1172 (s), 754 (m) cm $\mathrm{cm}^{-1}$.

MS $\left(\mathrm{ESI}^{+}\right) \mathrm{m} / \mathrm{z}(\%) 595\left([\mathrm{M}+\mathrm{Na}]^{+}\left[{ }^{81} \mathrm{Br}\right], 71\right), 593\left([\mathrm{M}+\mathrm{Na}]^{+}\left[{ }^{79} \mathrm{Br}\right], 72\right), 573\left([\mathrm{M}+1]^{+}\right.$ $\left.\left[{ }^{81} \mathrm{Br}\right], 99\right), 571\left([\mathrm{M}+1]+{ }^{+}\left[{ }^{79} \mathrm{Br}\right], 100\right), 529$ (24), 527 (24), 447 (18), 357 (8).

HRMS $\left(\right.$ ESI $^{+}$) calcd for $\mathrm{C}_{26} \mathrm{H}_{24}{ }^{81} \mathrm{BrN}_{2} \mathrm{O}_{6} \mathrm{~S}, 573.0512$ and $\mathrm{C}_{26} \mathrm{H}_{24}{ }^{79} \mathrm{BrN}_{2} \mathrm{O}_{6} \mathrm{~S}, 571.0533$; found 573.0504 and 571.0526 .

$[\alpha]_{\mathrm{D}}^{27}+184^{\circ}\left(c 0.27, \mathrm{CHCl}_{3}\right)$.

(2R,3aS,8aS)-8-Benzenesulfonyl-3a-bromo-3,3a,8,8a-tetrahydro-2H-pyrrolo[2,3-

$b$ ]indole-1,2-dicarboxylic acid dimethyl ester (6f).
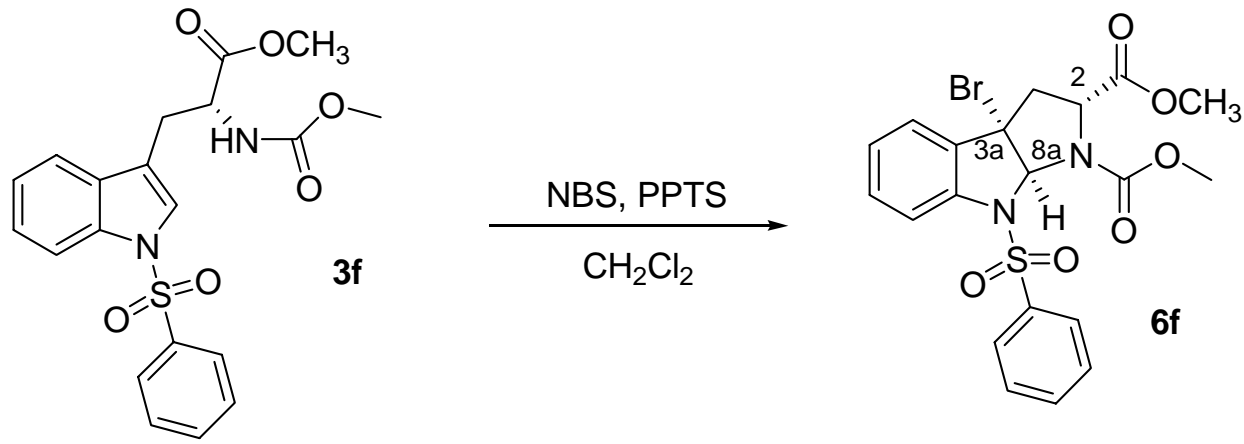
To a solution of the tryptophan derivative $3 f(100 \mathrm{mg}, 0.24 \mathrm{mmol})$ in $\mathrm{CH}_{2} \mathrm{Cl}_{2}(2 \mathrm{~mL})$ under argon was added $\mathrm{N}$-bromosuccinimide (43 $\mathrm{mg}, 0.24 \mathrm{mmol}, 1$ equiv) and pyridinium $p$-toluenesulfonate (61 $\mathrm{mg}, 0.24 \mathrm{mmol}, 1$ equiv). The resulting yellow solution was stirred at $25^{\circ} \mathrm{C}$ for 3 hours. The mixture was diluted with $\mathrm{CH}_{2} \mathrm{Cl}_{2}(8 \mathrm{~mL})$ and treated with a $10 \% \mathrm{NaHCO}_{3}$ aqueous solution $(10 \mathrm{~mL})$ and a $10 \% \mathrm{Na}_{2} \mathrm{~S}_{2} \mathrm{O}_{4}$ aqueous solution $(10 \mathrm{~mL})$. The organic layer was dried over $\mathrm{Na}_{2} \mathrm{SO}_{4}$ and the solvents were removed under reduced pressure. The residue was purified by flash chromatography (60:40, hexanes/EtOAc) to afford $99 \mathrm{mg}$ (83\%yield) as a colourless oil.

${ }^{1}$ H NMR (400 MHz, $\left.\mathrm{CDCl}_{3}\right) \delta$ 7.7-7.6 (m, 2H, ArH), 7.58 (d, $\left.J=8.1 \mathrm{~Hz}, 1 \mathrm{H}, \mathrm{ArH}\right)$, $7.46(\mathrm{t}, J=7.5 \mathrm{~Hz}, 1 \mathrm{H}, \mathrm{ArH})$ 7.4-7.3 (m, 3H, ArH), 7.3-7.2 (m, 1H, ArH), 7.17 (t, $J=$ $7.1 \mathrm{~Hz}, 1 \mathrm{H}, \mathrm{ArH}), 6.25$ (br, $1 \mathrm{H}, \mathrm{H} 8 \mathrm{a}), 3.9-3.7\left(\mathrm{~m}, 4 \mathrm{H}, \mathrm{H} 2+\mathrm{CO}_{2} \mathrm{CH}_{3}\right), 3.73(\mathrm{~s}, 3 \mathrm{H}$, $\left.\mathrm{CO}_{2} \mathrm{CH}_{3}\right), 3.08(\mathrm{dd}, J=12.7,5.9 \mathrm{~Hz}, 1 \mathrm{H}, \mathrm{H} 3 \mathrm{~A}), 2.78(\mathrm{dd}, J=12.7,10.5 \mathrm{~Hz}, 1 \mathrm{H}, \mathrm{H} 3 \mathrm{~B})$ ppm.

${ }^{13}$ C NMR (100 MHz, $\left.\mathrm{CDCl}_{3}\right) \delta 170.7$ (s, $\left.\underline{\mathrm{CO}}_{2} \mathrm{CH}_{3}\right), 154.3$ (s, OCON), 140.3 (s), 138.5 (s), 133.9 (s), 133.7(d), 131.2 (d), 129.0 (d, 2x), 128.2 (d), 126.6 (d), 124.1 (d, 2x), 119.0 (d), 86.8(d, C8a), 59.3(d, C2), 59.2(s, C3a), 53.2(q, $\left.\mathrm{OCH}_{3}\right), 52.9$ (q, $\left.\mathrm{OCH}_{3}\right), 43.5$ (t, C3) ppm.

IR (NaCl) v $3068(\mathrm{~m}, \mathrm{C}-\mathrm{H}), 3003(\mathrm{~m}, \mathrm{C}-\mathrm{H}), 2954(\mathrm{~s}, \mathrm{C}-\mathrm{H}), 1749$ (s, CO), 1714 (s, CO), 1600 (m), 1447 (s), 1366 (s), 1172 (s), 1091 (s), 731 (s) $\mathrm{cm}^{-1}$.

MS $\left(\mathrm{ESI}^{+}\right) \mathrm{m} / \mathrm{z}(\%) 519\left([\mathrm{M}+\mathrm{Na}]^{+}\left[{ }^{81} \mathrm{Br}\right], 100\right), 517\left([\mathrm{M}+\mathrm{Na}]^{+}\left[{ }^{79} \mathrm{Br}\right], 95\right), 383(10)$.

HRMS $\left(\right.$ ESI $^{+}$) calcd for $\mathrm{C}_{20} \mathrm{H}_{19}{ }^{81} \mathrm{BrN}_{2} \mathrm{NaO}_{6} \mathrm{~S}, 519.0018$ and $\mathrm{C}_{20} \mathrm{H}_{19}{ }^{79} \mathrm{BrN}_{2} \mathrm{O}_{6}, 517.0039$; found 519.0010 and 517.0031 .

$[\alpha]_{\mathrm{D}}^{28}+169^{\circ}\left(c 0.30, \mathrm{CHCl}_{3}\right)$. 
3. ${ }^{1} \mathrm{H}$ NMR, ${ }^{13} \mathrm{C}$ NMR Spectral Data and HPLC chromatograms


S-39 

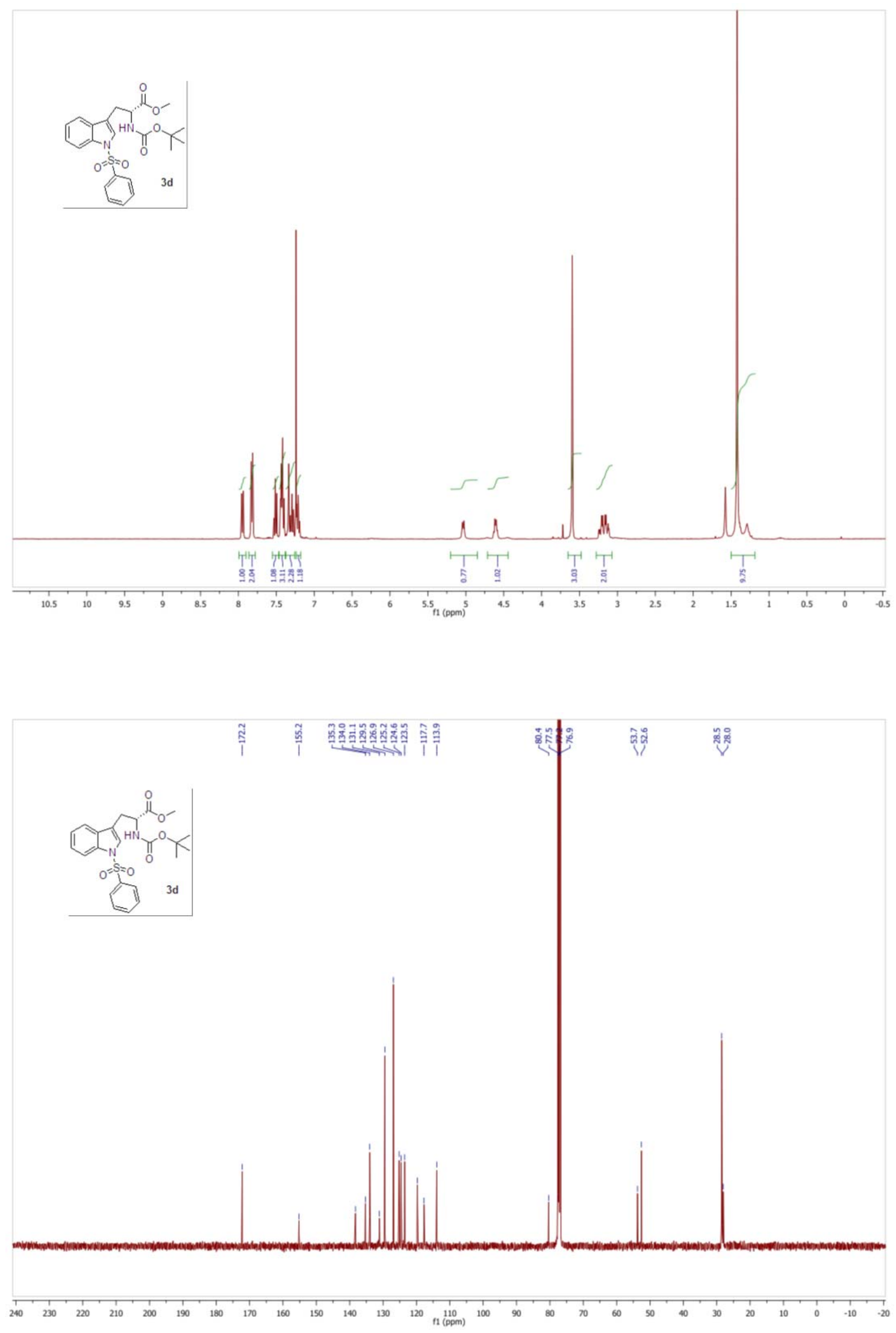

S-40 

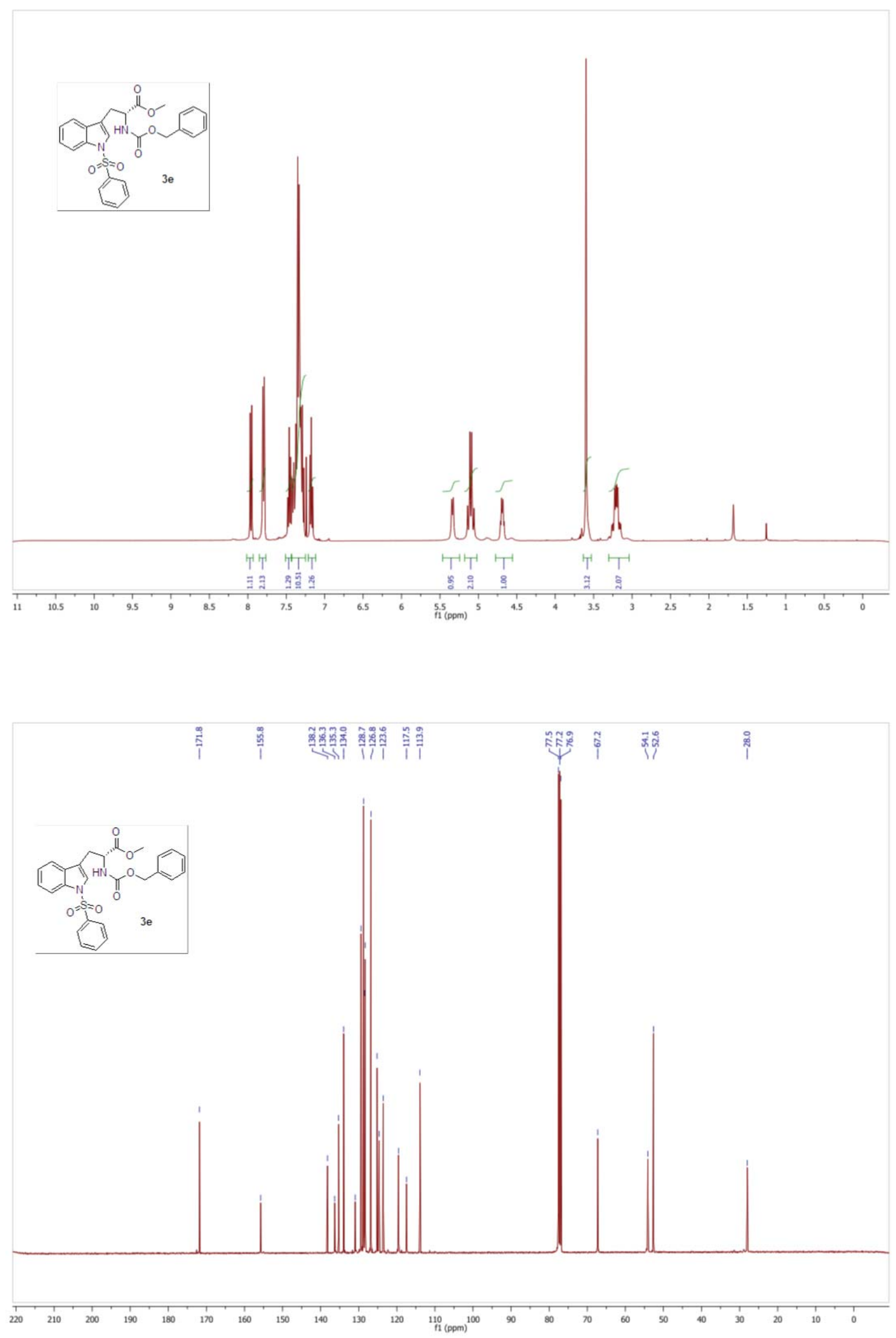

S-41 

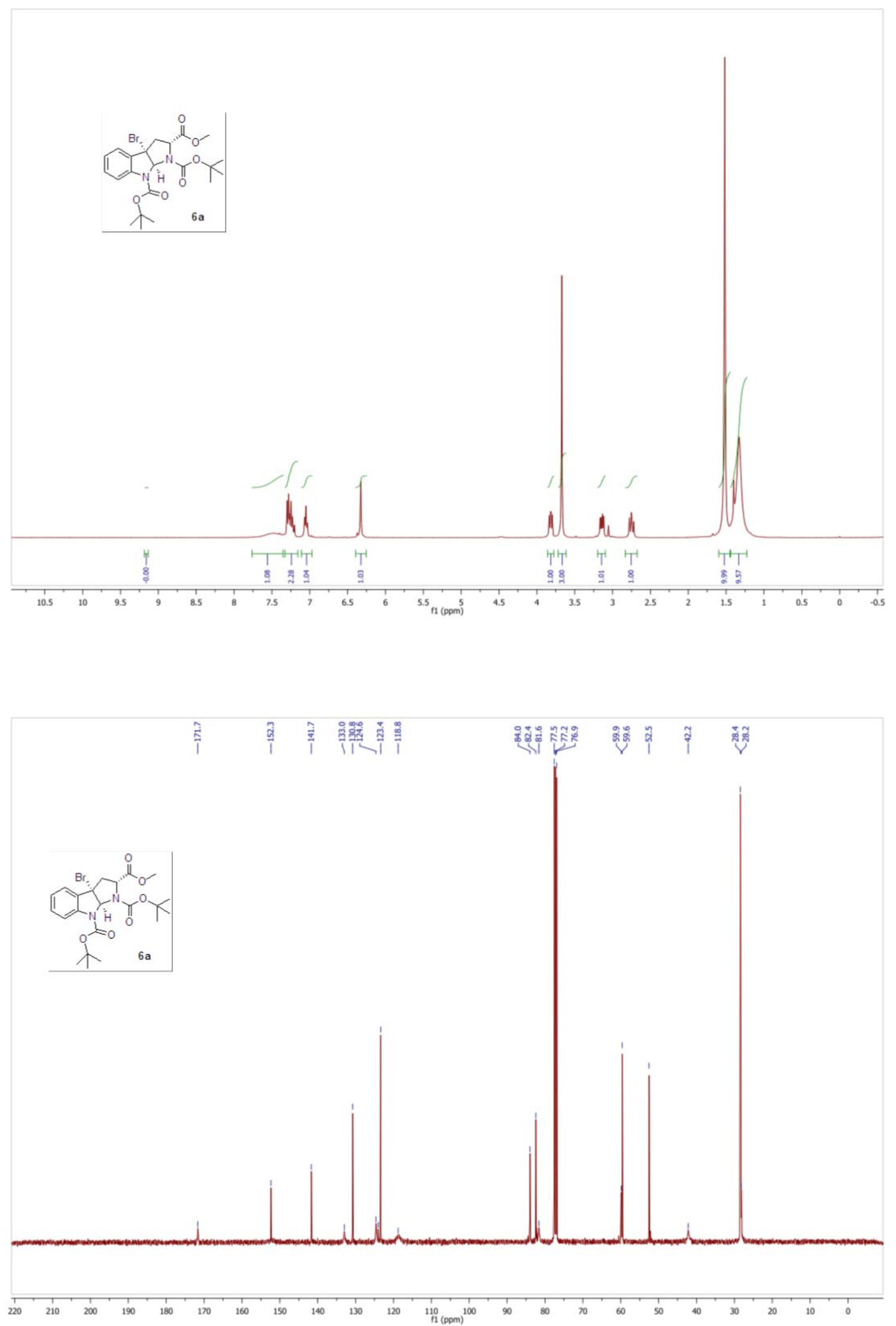
Hichrom Hypersil H5 25cm x 10 mm

Hexane/EtOAc:80/20, Flow: $3.0 \mathrm{~mL} / \mathrm{min}$

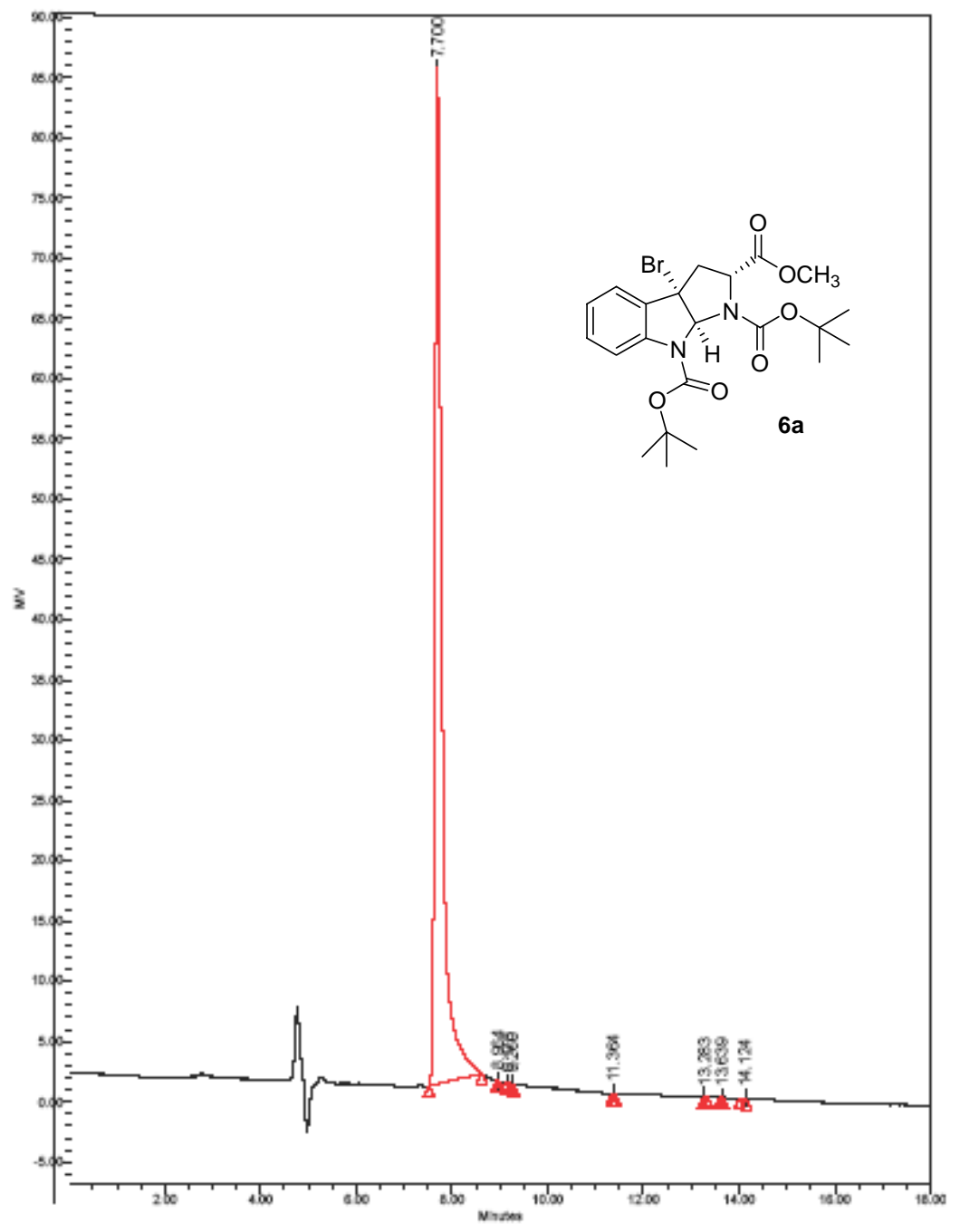

\begin{tabular}{|l|l|r|r|r|r|l|l|l|l|l|}
\hline & Name & $\begin{array}{c}\text { Retention Time } \\
(\mathrm{min})\end{array}$ & $\begin{array}{c}\text { Area } \\
\left(\mu \mathrm{V}^{*} \mathrm{sec}\right)\end{array}$ & $\%$ Area & $\begin{array}{c}\text { Height } \\
(\mu \mathrm{V})\end{array}$ & Int Type & Amount & Units & Peak Type & Peak Codes \\
\hline 1 & & 7.700 & 876791 & 99.89 & 84160 & BB & & & Unknown & \\
\hline 2 & & 8.964 & 70 & 0.01 & 48 & BB & & & Unknown & I19 \\
\hline 3 & & 9.178 & 145 & 0.02 & 59 & BB & & & Unknown & I19 \\
\hline 4 & & 9.260 & 98 & 0.01 & 51 & BB & & & Unknown & I19 \\
\hline 5 & & 11.364 & 117 & 0.01 & 62 & B B & & & Unknown & I19 \\
\hline 6 & & 13.283 & 108 & 0.01 & 78 & BB & & & Unknown & I19 \\
\hline 7 & & 13.639 & 157 & 0.02 & 65 & BB & & & Unknown & I19 \\
\hline 8 & & 14.124 & 262 & 0.03 & 70 & BB & & & Unknown & I19 \\
\hline
\end{tabular}



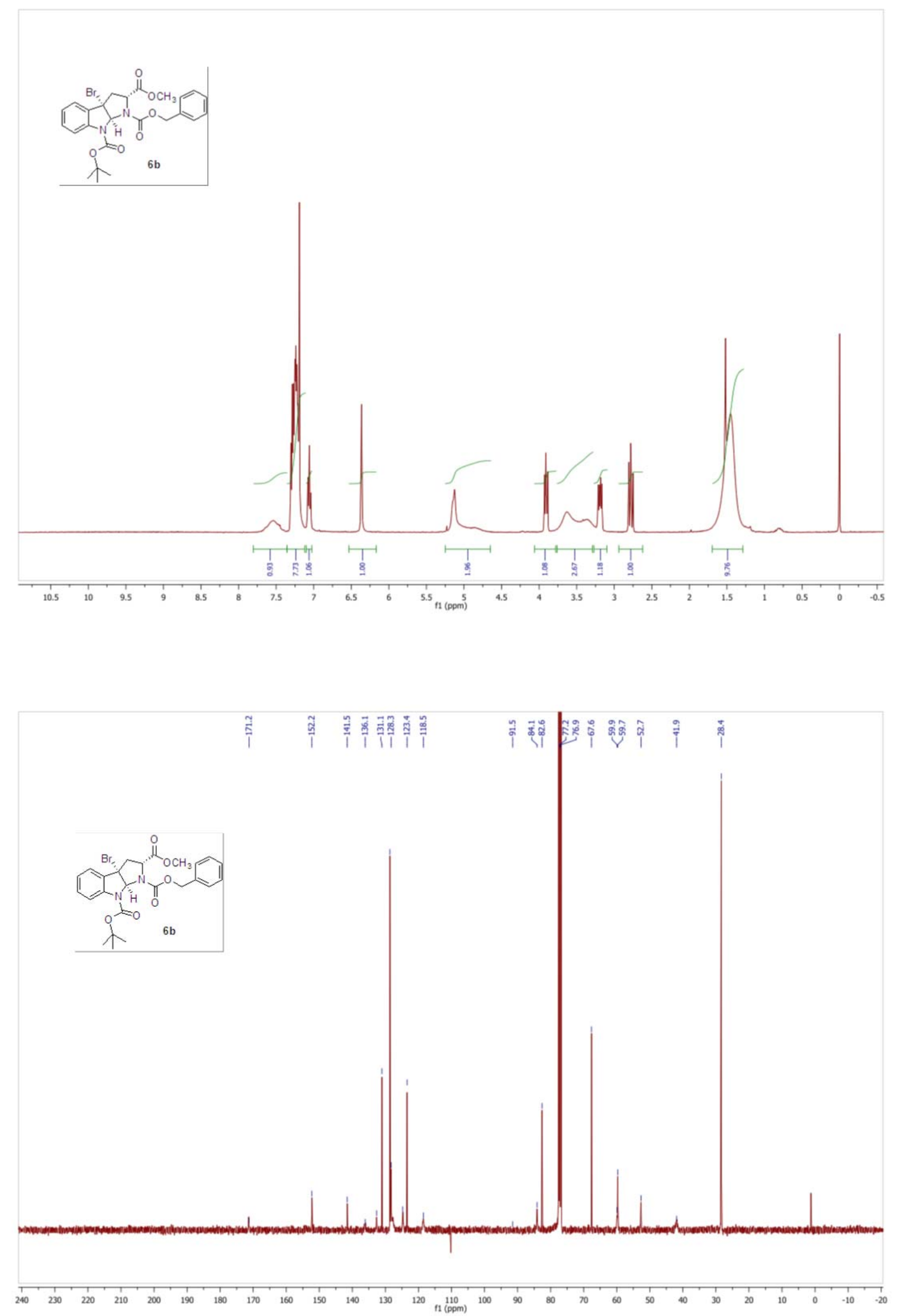
Hichrom Hypersil H5 25cm x 10 mm

Hexane/EtOAc:80/20, Flow: $3.0 \mathrm{~mL} / \mathrm{min}$

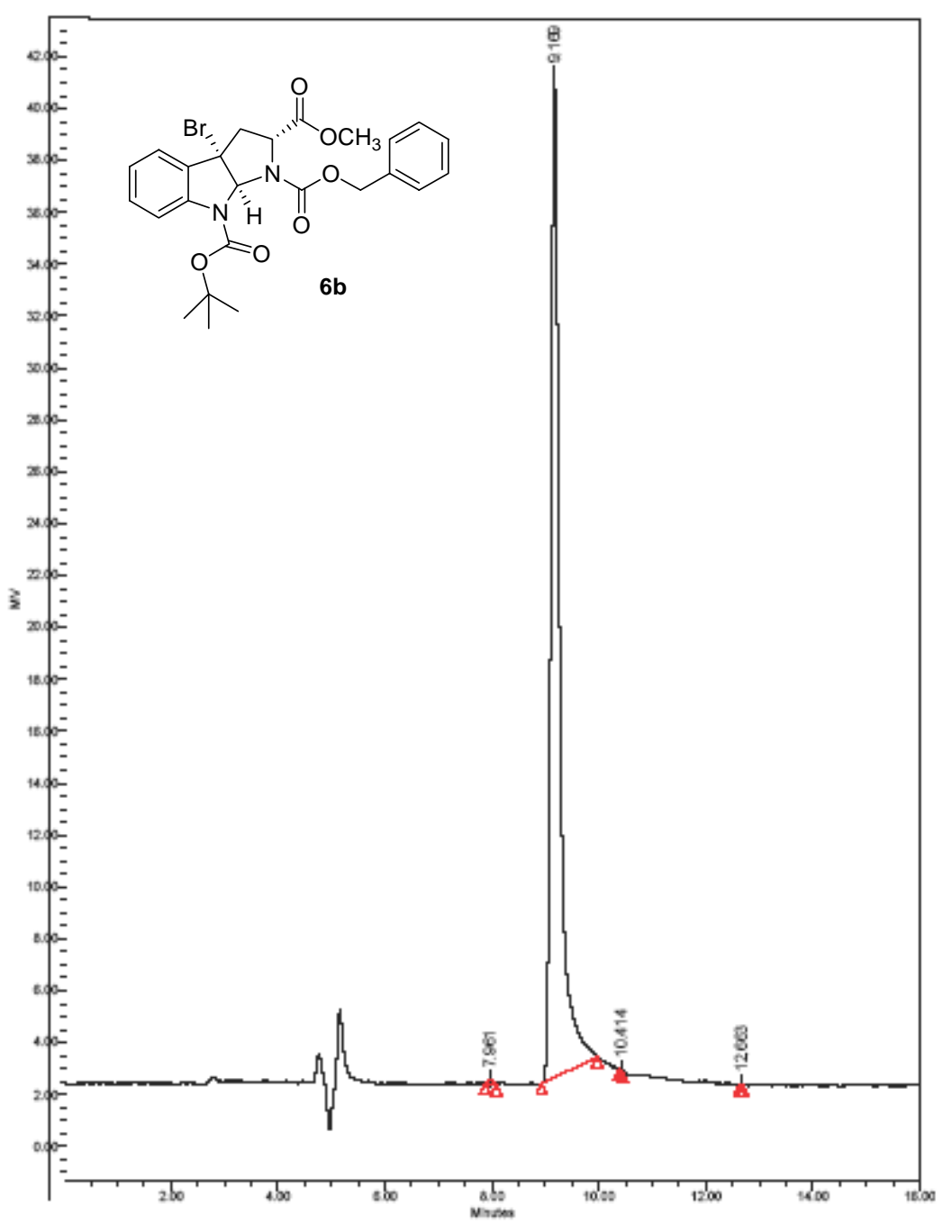

\begin{tabular}{|l|l|r|r|r|r|l|l|l|l|l|}
\hline & Name & $\begin{array}{c}\text { Retention Time } \\
(\mathrm{min})\end{array}$ & $\begin{array}{c}\text { Area } \\
\left(\mu \mathrm{V}^{\star} \mathrm{sec}\right)\end{array}$ & $\%$ Area & $\begin{array}{c}\text { Height } \\
(\mu \mathrm{V})\end{array}$ & Int Type & Amount & Units & Peak Type & Peak Codes \\
\hline 1 & & 7.961 & 1194 & 0.26 & 170 & BB & & & Unknown & \\
\hline 2 & & 9.169 & 450501 & 99.68 & 38715 & BB & & & Unknown & \\
\hline 3 & & 10.414 & 96 & 0.02 & 63 & BB & & & Unknown & I19 I38 \\
\hline 4 & & 12.663 & 164 & 0.04 & 73 & BB & & & Unknown & I19 \\
\hline
\end{tabular}



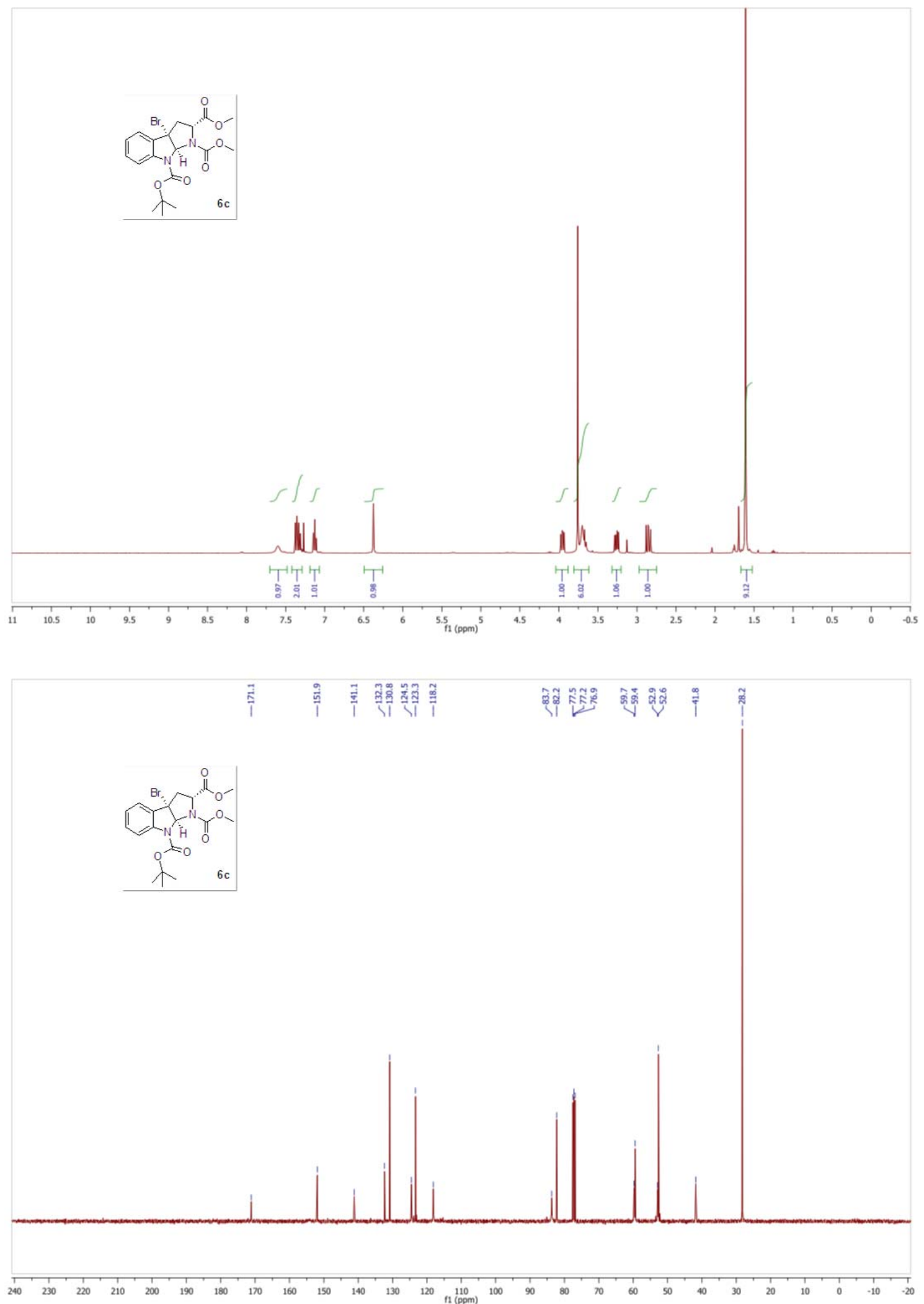
Hichrom Hypersil H5 25cm x 10 mm

Hexane/EtOAc:70/30, Flow: $3.0 \mathrm{~mL} / \mathrm{min}$

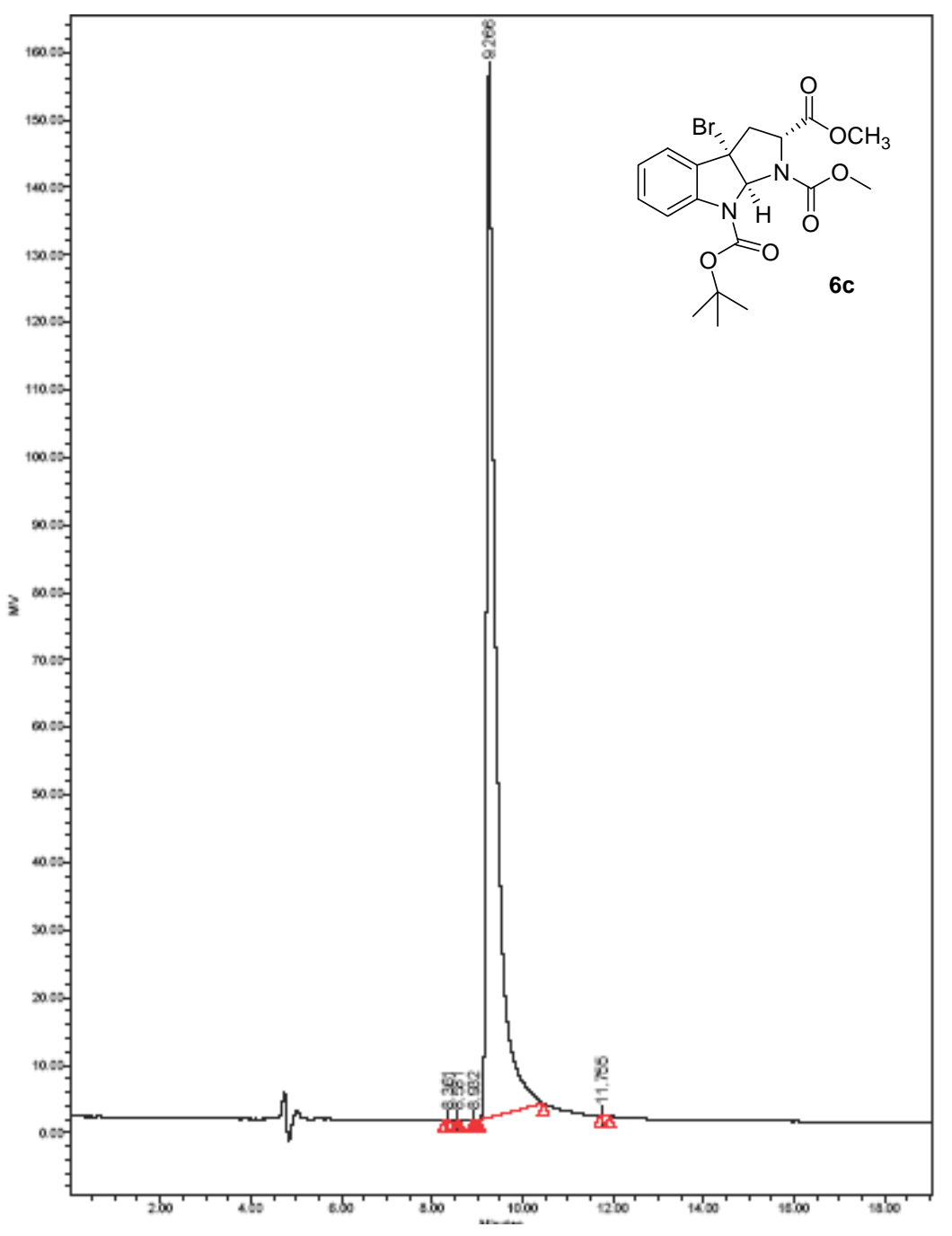

\begin{tabular}{|l|l|r|r|r|r|l|l|l|l|l|}
\hline & Name & $\begin{array}{c}\text { Retention Time } \\
(\mathrm{min})\end{array}$ & $\begin{array}{c}\text { Area } \\
\left(\mu \mathrm{V}^{\star} \mathrm{sec}\right)\end{array}$ & $\begin{array}{c}\% \text { Area } \\
(\mu \mathrm{V})\end{array}$ & $\begin{array}{c}\text { Height } \\
\text { Int Type }\end{array}$ & Amount & Units & Peak Type & Peak Codes \\
\hline 1 & & 8.361 & 229 & 0.01 & 54 & BB & & & Unknown & \\
\hline 2 & & 8.581 & 126 & 0.01 & 57 & BB & & & Unknown & I19 \\
\hline 3 & & 8.932 & 80 & 0.00 & 47 & BB & & & Unknown & I19 \\
\hline 4 & & 9.266 & 2269623 & 99.94 & 155283 & BB & & & Unknown & \\
\hline 5 & & 11.755 & 866 & 0.04 & 86 & BB & & & Unknown & I19 \\
\hline
\end{tabular}



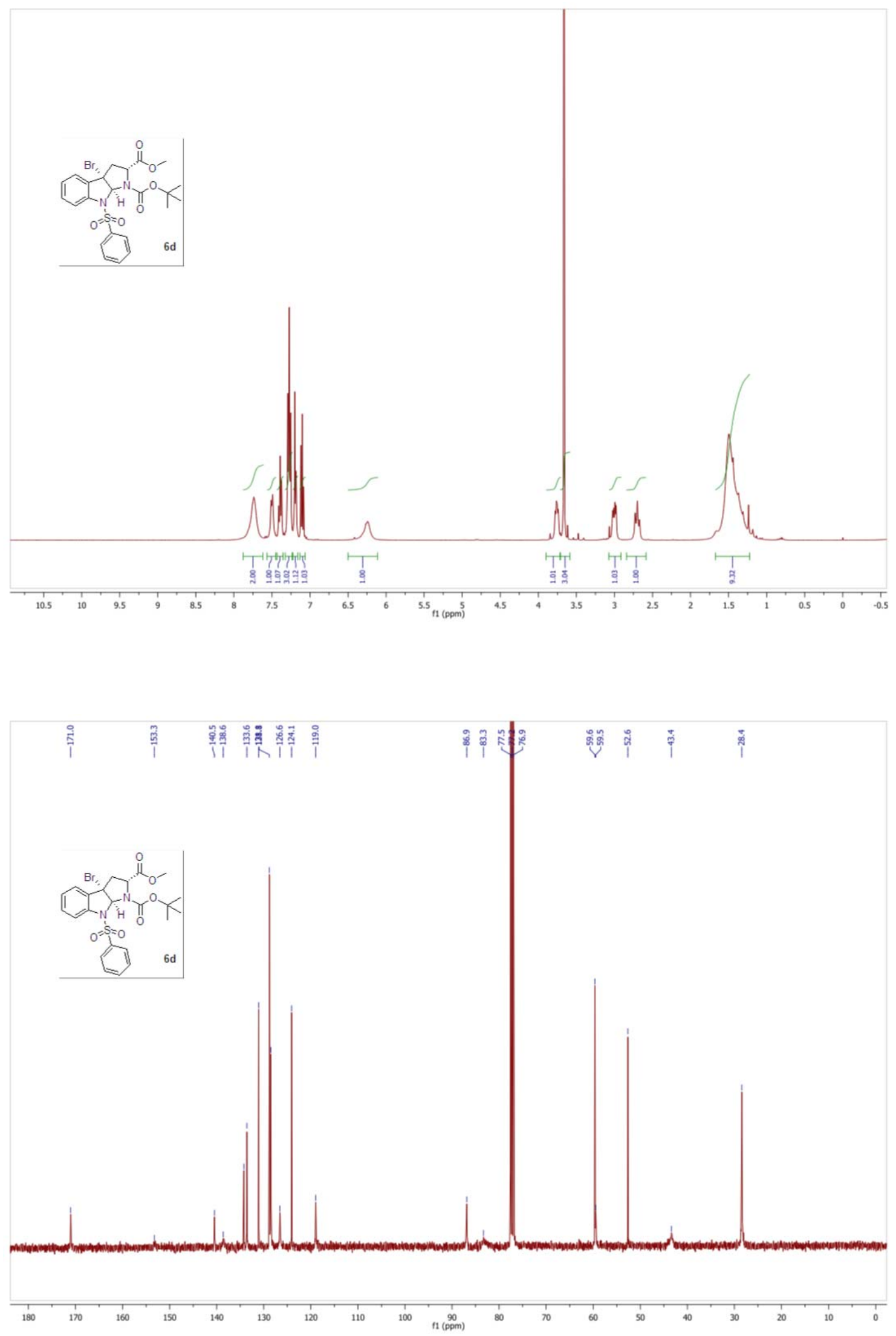

S-48 
Hichrom Hypersil H5 25cm x 10 mm

Hexane/EtOAc: 70/30, Flow: $3.0 \mathrm{~mL} / \mathrm{min}$

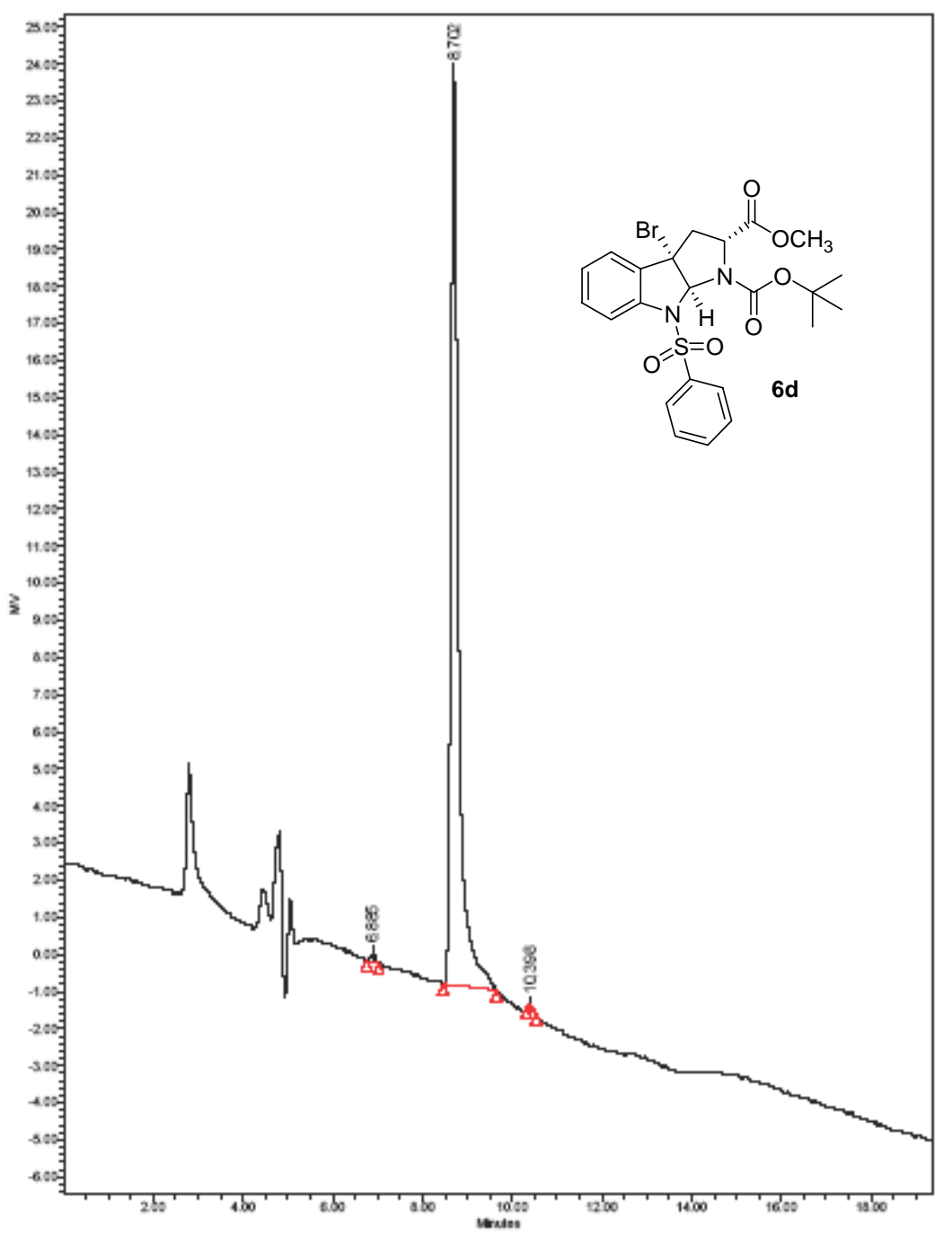

\begin{tabular}{|l|l|r|r|r|r|l|l|l|l|l|}
\hline & Name & $\begin{array}{c}\text { Retention Time } \\
(\mathrm{min})\end{array}$ & $\begin{array}{c}\text { Area } \\
\left(\mu \mathrm{V}^{*} \mathrm{sec}\right)\end{array}$ & $\%$ Area & $\begin{array}{c}\text { Height } \\
(\mu \mathrm{V})\end{array}$ & Int Type & Amount & Units & Peak Type & Peak Codes \\
\hline 1 & & 6.885 & 1788 & 0.60 & 206 & BB & & & Unknown & I21 I19 \\
\hline 2 & & 8.702 & 295025 & 99.13 & 24665 & Bb & & & Unknown & \\
\hline 3 & & 10.398 & 805 & 0.27 & 130 & bb & & & Unknown & \\
\hline
\end{tabular}



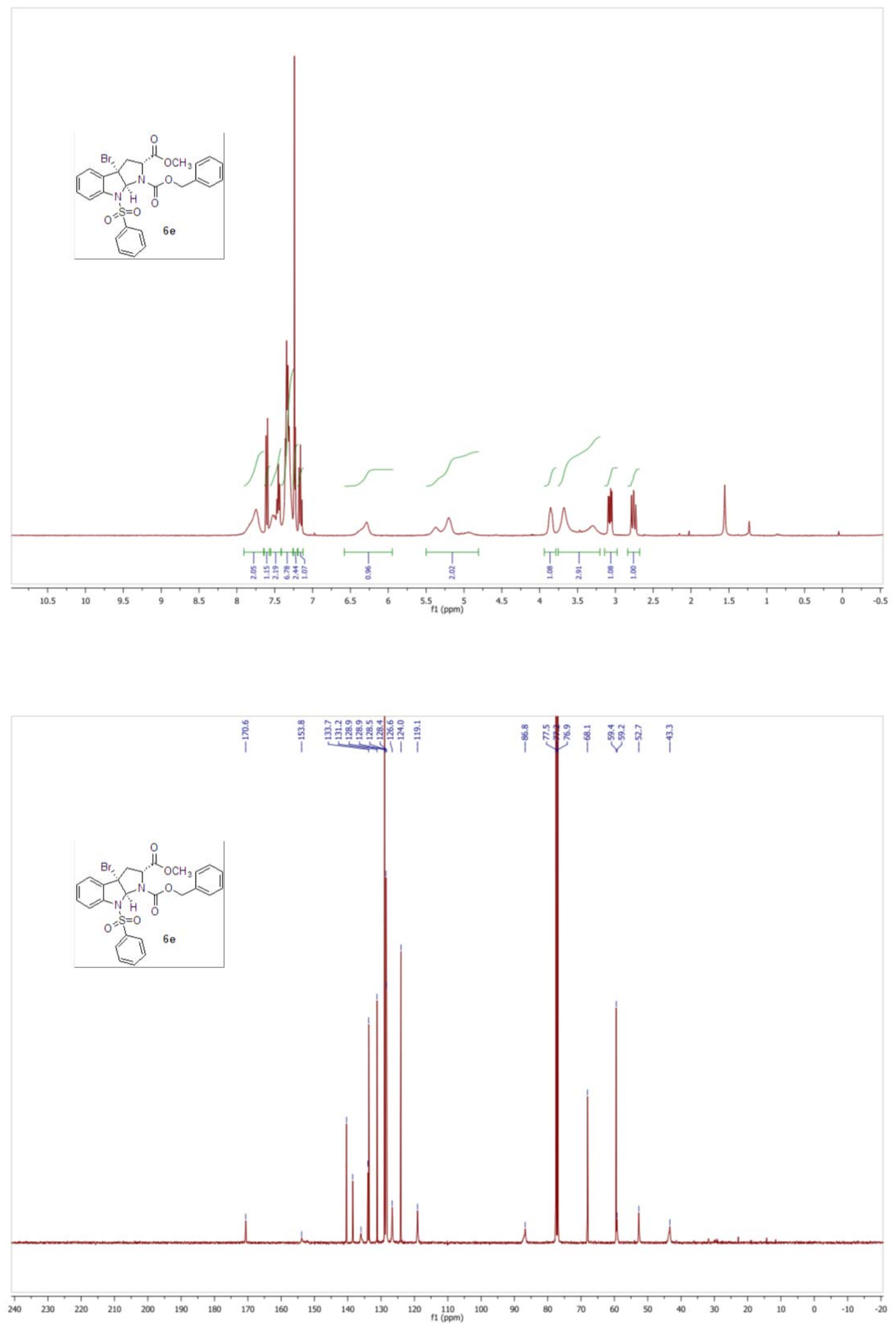
Hichrom Hypersil H5 25cm x 10 mm

Hexane/EtOAc: 70/30, Flow: $3.0 \mathrm{~mL} / \mathrm{min}$

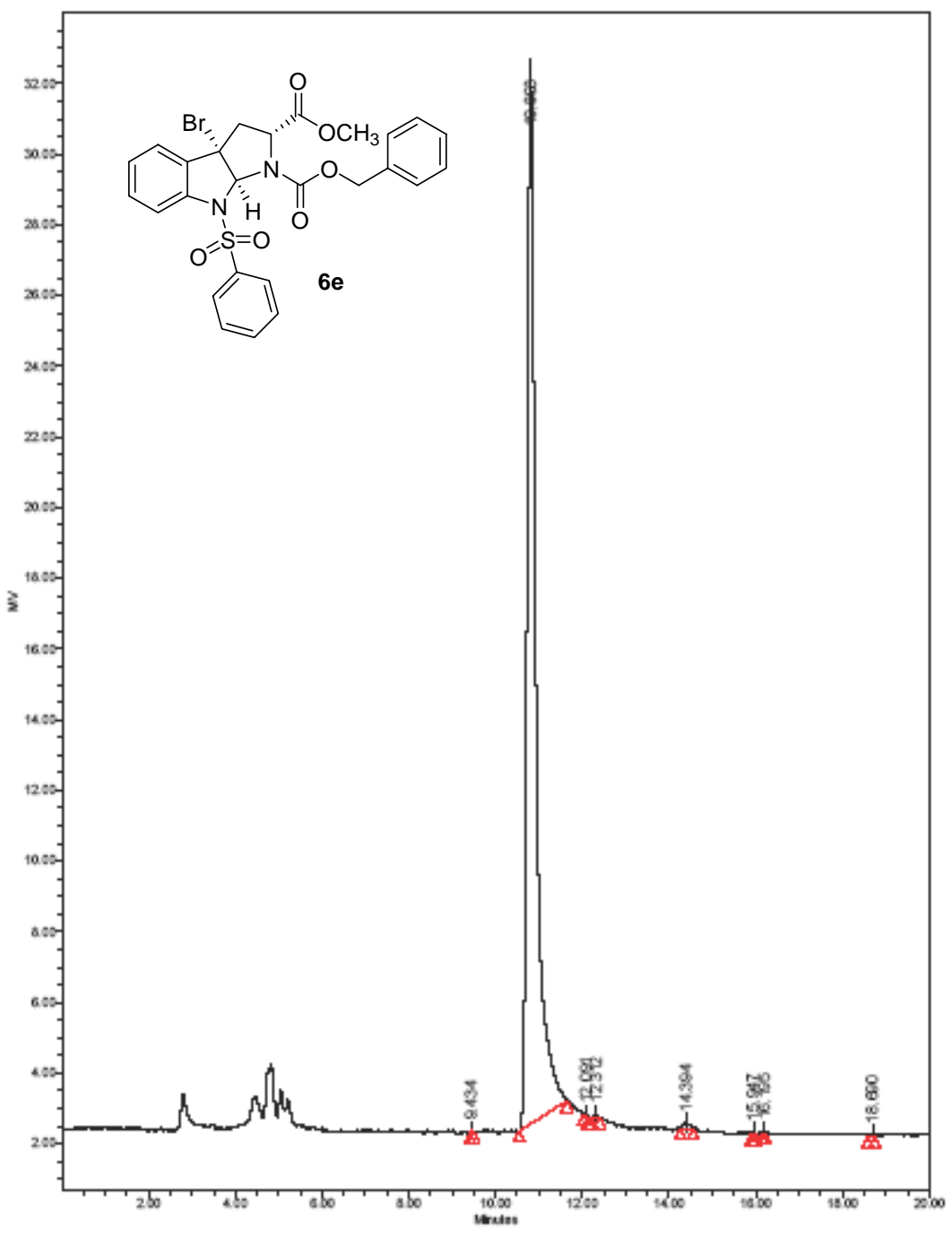

\begin{tabular}{|l|l|r|r|r|r|l|l|l|l|l|}
\hline & Name & $\begin{array}{c}\text { Retention Time } \\
(\mathrm{min})\end{array}$ & $\begin{array}{c}\text { Area } \\
\left(\mu \mathrm{V}^{*} \mathrm{sec}\right)\end{array}$ & $\%$ Area & $\begin{array}{c}\text { Height } \\
(\mu \mathrm{V})\end{array}$ & Int Type & Amount & Units & Peak Type & Peak Codes \\
\hline 1 & & 9.434 & 154 & 0.04 & 47 & BB & & & Unknown & \\
\hline 2 & & 10.808 & 426234 & 99.22 & 30039 & BB & & & Unknown & \\
\hline 3 & & 12.091 & 234 & 0.05 & 55 & BB & & & Unknown & \\
\hline 4 & & 12.312 & 566 & 0.13 & 98 & BB & & & Unknown & \\
\hline 5 & & 14.394 & 1662 & 0.39 & 174 & BB & & & Unknown & \\
\hline 6 & & 15.947 & 131 & 0.03 & 60 & BB & & & Unknown & I19 \\
\hline 7 & & 16.195 & 126 & 0.03 & 46 & BB & & & Unknown & \\
\hline 8 & & 18.690 & 480 & 0.11 & 81 & BB & & & Unknown & I21 I19 \\
\hline
\end{tabular}



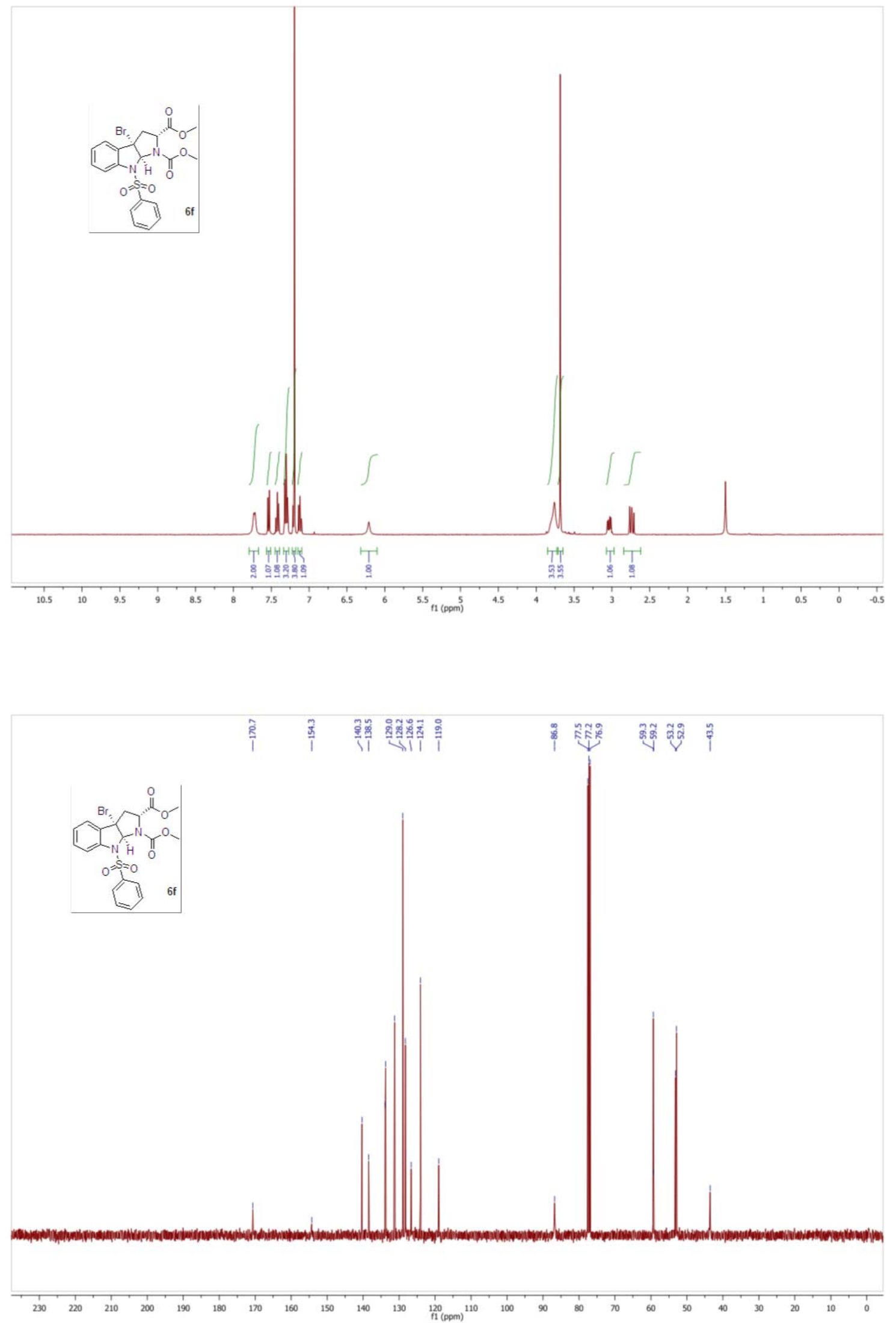
Hichrom Hypersil H5 25cm x 10 mm

Hexane/EtOAc: 70/30, Flow: $3.0 \mathrm{~mL} / \mathrm{min}$

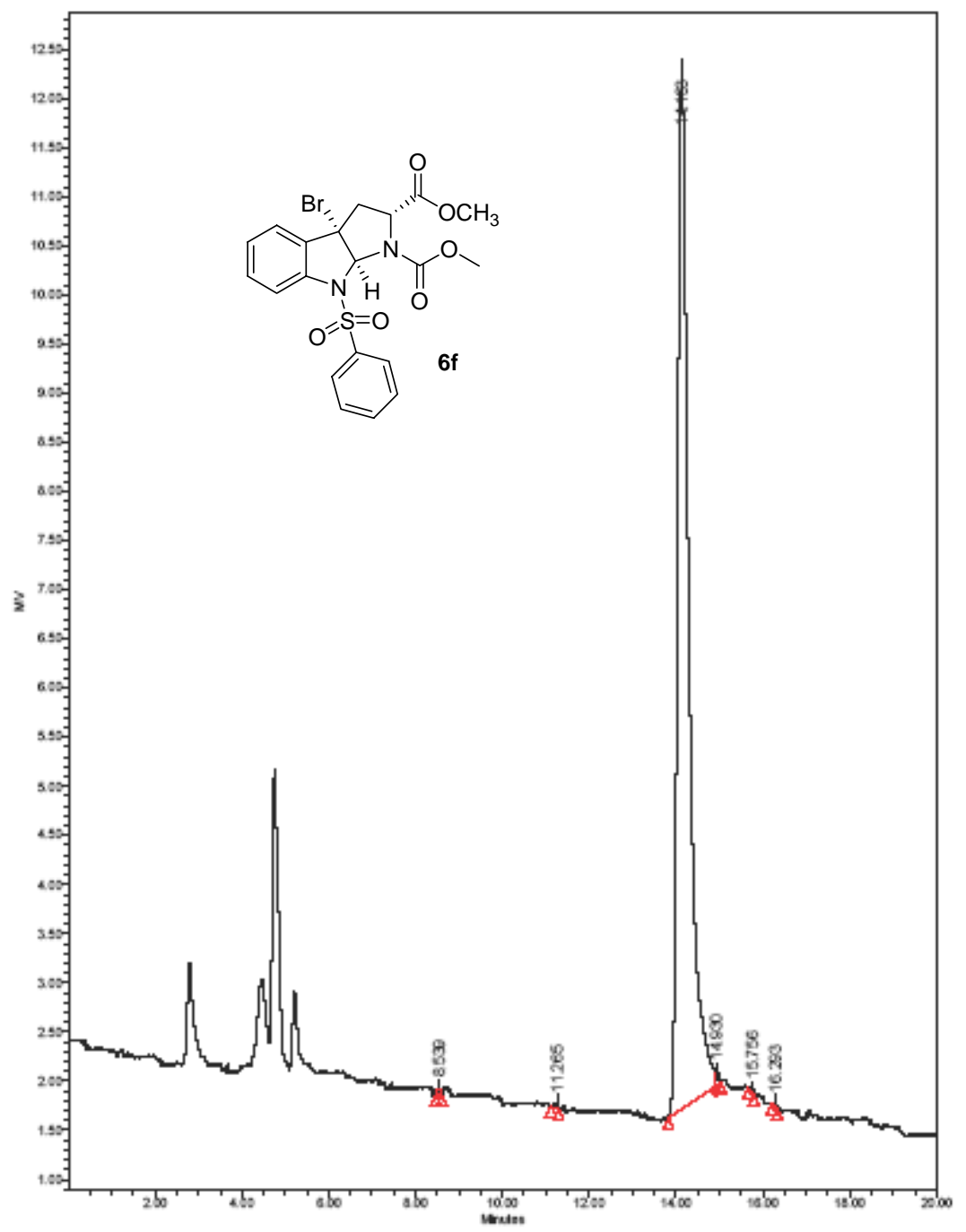

\begin{tabular}{|l|l|r|r|r|r|l|l|l|l|l|}
\hline Name & $\begin{array}{c}\text { Retention Time } \\
(\mathrm{min})\end{array}$ & $\begin{array}{c}\text { Area } \\
\left(\mu \mathrm{V}^{\star} \mathrm{sec}\right)\end{array}$ & $\%$ Area & $\begin{array}{c}\text { Height } \\
(\mu \mathrm{V})\end{array}$ & Int Type & Amount & Units & Peak Type & Peak Codes \\
\hline 1 & & 8.539 & 391 & 0.20 & 67 & BB & & & Unknown & \\
\hline 2 & & 11.265 & 286 & 0.15 & 58 & BB & & & Unknown & I06 I21 I19 \\
\hline 3 & & 14.133 & 193826 & 99.12 & 10596 & BV & & & Unknown & \\
\hline 4 & & 14.930 & 598 & 0.31 & 143 & VB & & & Unknown & I19 \\
\hline 5 & & 15.756 & 229 & 0.12 & 37 & BB & & & Unknown & \\
\hline 6 & & 16.293 & 208 & 0.11 & 44 & BB & & & Unknown & \\
\hline
\end{tabular}

